\title{
The impact of semantic impairment on verbal short-term memory in stroke aphasia and semantic dementia: A comparative study
}

\author{
Elizabeth Jefferies ${ }^{\text {a,* }}$, Paul Hoffman ${ }^{\text {a }}$, Roy Jones ${ }^{\mathrm{b}}$, Matthew A. Lambon Ralph ${ }^{\text {a }}$ \\ a Neuroscience and Aphasia Research Unit (NARU), Zochonis Building, School of Psychological Sciences, University of Manchester, \\ Oxford Road, Manchester M13 9PL, UK \\ b Research Institute for the Care of the Elderly, Bath, UK
}

Received 16 May 2006; revision received 5 June 2007

Available online 6 August 2007

\begin{abstract}
This study presents the first direct comparison of immediate serial recall in semantic dementia (SD) and transcortical sensory aphasia (TSA). Previous studies of the effect of semantic impairment on verbal short-term memory (STM) have led to important theoretical advances. However, different conclusions have been drawn from these two groups. This research aimed to explain these inconsistencies. We observed (a) qualitative differences between SD and TSA in the nature of the verbal STM impairment and (b) considerable variation within the TSA group. The SD and TSA patients all had poor semantic processing and good phonology. Reflecting this, both groups remained sensitive to phonological similarity and showed a reduced effect of lexicality in immediate serial recall. The SD patients showed normal serial position effects; in contrast, the TSA patients had poor recall of the initial list items and exhibited large recency effects on longer lists. The error patterns of the two groups differed: the SD patients made numerous phoneme migration errors whereas the TSA group were more likely to produce entire words in the wrong order, often initiating recall with terminal list items. The SD cases also showed somewhat larger effects of word frequency and imageability. We propose that these contrasting performance patterns are explicable in terms of the nature of the underlying semantic impairment. SD is associated with anterior lobe atrophy and produces degradation of semantic knowledge - this is more striking for less frequent/imageable items, accentuating the effects of these lexical/semantic variables in STM. SD patients frequently recombine the phonemes of different list items due to the reduced semantic constraint upon phonology (semantic binding: Patterson, K., Graham, N., \& Hodges, J. R. (1994). The impact of semantic memory loss on phonological representations. Journal of Cognitive Neuroscience, 6, 57-69). In contrast, the semantic impairment in TSA follows frontal or temporoparietal lesions and is associated with poor executive control of semantic processing (deregulated semantic cognition: Jefferies, E., \& Lambon Ralph, M. A. (2006). Semantic impairment in stroke aphasia vs. semantic dementia: a case-series comparison. Brain, 129, 2132-2147), explaining why these patients are liable to recall entire words out of serial order.
\end{abstract}

(C) 2007 Elsevier Inc. All rights reserved.

Keywords: Verbal short-term memory; Semantic dementia; Aphasia; Stroke; Semantic memory

\footnotetext{
* Corresponding author. Fax: +44 (0) 1612752873.

E-mail address: beth.jefferies@manchester.ac.uk (E. Jefferies).
} 


\section{Introduction}

Verbal STM is supported by multiple levels of representations utilised in language production and comprehension, including long-term knowledge of the sounds and meanings of familiar words. This lexical/semantic contribution explains why normal immediate serial recall (ISR) is better for (1) words vs. nonwords (e.g., Brener, 1940; Hulme, Maughan, \& Brown, 1991; referred to as the effect of "lexicality" below); (2) high compared with low frequency words (Gregg, Freedman, \& Smith, 1989; Hulme et al., 1997; Roodenrys \& Quinlan, 2000; Watkins \& Watkins, 1977); (3) imageable/ concrete words vs. abstract words (Bourassa \& Besner, 1994; Walker \& Hulme, 1999); and (4) semantically grouped vs. ungrouped words (Poirier \& Saint-Aubin, 1995; Saint-Aubin \& Poirier, 1999).

Neuropsychological studies have provided a particularly striking demonstration of the importance of word meaning in verbal STM. Patients with both stroke aphasia and semantic dementia (SD) have been used to study the effect of semantic impairment on ISR. However, investigations of these two groups have proceeded in parallel and have focussed on different issues, giving rise to distinct theories about the way in which long-term linguistic knowledge contributes to verbal STM (e.g., Martin \& Saffran, 1997; Patterson, Graham, \& Hodges, 1994).

Martin and Saffran (1997) examined 15 stroke aphasic patients with semantic and/or phonological processing impairments. Poor semantic processing was associated with reduced effects of imageability in ISR, whereas poor phonological processing was associated with increased imageability effects (correlations with word frequency largely failed to reach significance). Semantic and phonological deficits also had differential effects on the shape of the serial position curve: the semantically impaired stroke aphasic patients showed poorer recall of items at the beginning of lists (reduced primacy effect), whereas the phonologically impaired patients showed poorer recall of items toward the end of the lists (reduced recency effect; see also Martin \& Saffran, 1990).

These findings were interpreted within Dell and O'Seaghda's (1992) interactive activation (IA) model of word production. By this view, verbal STM results from temporary activation within phonological, lexical and semantic nodes (see also Martin \& Ayala, 2004; Martin \& Gupta, 2004). The maintenance of this activation is dependant on interactions between these processing levels-patients with lexical-semantic deficits have diminished semantic feedback to the phonological layer and so do not show the normal ISR advantage for imageable words, while those with phonological problems rely on this feedback more strongly and show enhanced imageability effects. Serial position effects can also be explained within this model: the initial words in a list accumulate the greatest amount of semantic feedback before recall because it takes time for activation to spread from the phonological units to the semantic units and back again. Items towards the end of lists receive less semantic support but their recall is boosted by the fact that activation in the phonological units has less time to decay.

Martin and colleagues also suggested that the phonological and semantic contributions to verbal STM are dissociable in stroke aphasia (Martin \& Lesch, 1996; Martin, Lesch, \& Bartha, 1999; Martin, Shelton, \& Yaffee, 1994). These studies found similar results to those described above. Patients with semantic STM problems (as revealed by poor category probe performance but relatively intact functioning on a similar rhyme probe task) showed normal effects of phonological similarity and recency but a reduced influence of lexicality and primacy in ISR, whereas patients with poor retention of phonological information displayed the opposite pattern (Martin et al., 1994). These deficits were argued to reflect damage to semantic and phonological STM stores. Recent research has suggested that a failure of inhibition may lie at the heart of the semantic STM deficit (Hamilton \& Martin, 2005).

Studies of verbal STM in patients with SD have had a rather different focus. Rather than concentrating on the influence of lexical/semantic variables and serial position effects in ISR, this research has explored the way in which word meaning supports phonological coherence. SD causes a highly specific degradation of amodal conceptual representations impairing performance across verbal and nonverbal domains (Bozeat, Lambon Ralph, Patterson, Garrard, \& Hodges, 2000). Other aspects of language and cognition are spared (Hodges, Patterson, Oxbury, \& Funnell, 1992; Snowden, Goulding, \& Neary, 1989). SD patients produce fluent, well-formed sentences and continue to perform well on phonological tasks (Jefferies, Jones, Bateman, \& Lambon Ralph, 2005; Jefferies, Patterson, Bateman, Jones, \& Lambon Ralph, 2006). However, they make frequent phoneme order errors in ISR and consequently their word span is severely impaired. Phonemes typically migrate to the same syllable position in a neighbouring word, for example, "mint, rug" $\rightarrow$ "rint, mug" (Patterson et al., 1994). This phonological breakdown is more severe for words that are poorly understood compared with those that are still comprehended relatively well, even when word frequency is matched (Jefferies, Jones, Bateman, \& Lambon Ralph, 2004; Jefferies et al., 2005; Jefferies, Patterson, Jones, Bateman, \& Lambon Ralph, 2004a; Knott, Patterson, \& Hodges, 1997, 2000). This strong association between semantic impairment and phonological disintegration in ISR led Patterson et al. (1994) to suggest that there are two sources of constraint on phonology. First, because the elements of 
a word are always activated together, they become associated in the phonological system which can then develop pattern completion properties. In addition, every time a word is spoken or comprehended, semantic activation co-occurs with phonological activation. Consequently, semantic-phonological interaction provides another strong constraint on phonological coherence (referred to as "semantic binding" below; see also Jefferies, Frankish, \& Lambon Ralph, 2006).

These theories all suggest a close relationship between verbal STM and speech processing. The IA and "semantic binding" hypotheses both propose that verbal STM is underpinned by phonological and semantic representations that are employed in language processing more generally. They also agree that there is strong interactivity between phonology and semantics. There is an implicit assumption that "semantic impairment" in SD and stroke aphasia should produce similar patterns of deficits on ISR tasks (see Martin \& Saffran, 1997). However, a review of the existing literature suggests that there may be some differences in the way that semantic impairment affects ISR in SD and stroke aphasia (see Table 1) which are problematic for the theoretical accounts discussed above.

(1) Aphasic patients with semantic impairment show weakened effects of lexical/semantic variables in ISR, as predicted by the IA model (Martin \& Saffran, 1997). In contrast, although SD patients show reduced effects of lexicality on ISR (Jefferies et al., 2005), they show robust effects of word frequency (the evidence for imageability is more mixed; Knott et al., 1997; Knott, Patterson, \& Hodges, 2000; Majerus, Norris, \& Patterson, 2007; McCarthy \& Warrington, 2001; Reilly, Martin, \& Grossman, 2005).

(2) The semantic deficits in SD and aphasia have different effects on the shape of the serial position curve. Semantic impairment in aphasia is associated with poorer recall of the initial portion of the list, in line with the IA model (Martin \& Saffran, 1997). In contrast, SD patients have either relatively normal serial position curves characterised by both primacy and

Table 1

The impact of comprehension impairment on immediate serial recall in semantic dementia and stroke aphasia

\begin{tabular}{lcl}
\hline & Semantic dementia & Stroke aphasia \\
\hline $\begin{array}{l}\text { Lexicality effect } \\
\text { Frequency effect }\end{array}$ & $\begin{array}{l}\text { Reduced } \\
\text { Increased }\end{array}$ & $\begin{array}{l}\text { Reduced } \\
\text { Reduced or } \\
\text { unchanged }\end{array}$ \\
Imageability effect & $\begin{array}{l}\text { Increased or } \\
\text { unchanged }\end{array}$ \\
$\begin{array}{l}\text { Phoneme migrations } \\
\text { Shape of serial } \\
\text { position curve }\end{array}$ & $\begin{array}{l}\text { Increased } \\
\text { Unchanged }\end{array}$ & $\begin{array}{l}\text { Unknown } \\
\text { Reduced } \\
\text { primacy effect }\end{array}$ \\
\hline
\end{tabular}

recency effects or, for more semantically impaired patients, negligible recency effects despite substantial primacy effects (Jefferies, Jones et al., 2004; Knott et al., 1997). ${ }^{1}$ SD patients also show parallel serial position curves for "known" and "semantically degraded" words (Jefferies, Jones et al., 2004; Knott et al., 1997). These findings challenge the view that word meaning disproportionately influences the initial part of the serial position curve.

(3) According to the semantic binding hypothesis, word meaning helps to prevent phonemes from breaking apart and recombining with the elements of other list items (Patterson et al., 1994). In contrast, the IA model does not propose a special role for word meaning in preventing phoneme migrations in ISR - rather, feedback from semantics helps to overcome rapid phonological decay (Martin \& Saffran, 1997). Although SD patients make frequent phoneme migration errors when recalling lists of semantically degraded words, it is not clear if semantic impairment in aphasia reliably produces phoneme migrations in ISR.

In summary, the AI and semantic binding hypotheses both seek to explain the effect of semantic impairment on verbal STM but they make different predictions and receive support from different patient groups. We propose that these contrasting theories reflect the diverse ways in which semantic cognition is impaired in stroke aphasia and SD (Jefferies \& Lambon Ralph, 2006). Comprehension problems in these groups arise from different areas of brain damage: SD cases have focal atrophy of the inferior, anterior temporal lobes bilaterally (Mummery et al., 2000; Nestor, Fryer, \& Hodges, 2006), while semantic impairment in stroke aphasia follows infarcts in either temporoparietal or prefrontal regions in the left hemisphere (e.g., Berthier, 2001; Chertkow, Bub, Deaudon, \& Whitehead, 1997).

One way in which the semantic deficit might differ between these groups was suggested by Martin and Gupta (2004): poor comprehension in stroke aphasia might reflect rapid decay within semantic-lexical connections, rather than degradation of conceptual knowledge itself (as in SD). Although aphasic patients with lexical and/or phonological problems might be specifically impaired at verbal semantic tasks, many stroke aphasic patients (including the patients with transcortical sensory aphasia/TSA studied here) fail both verbal and nonverbal semantic tasks (e.g., De Renzi, Faglioni,

\footnotetext{
${ }^{1}$ Reilly et al. (2005) observed negligible primacy effects in two of their five SD cases and more normal serial position curves for the remaining three patients. The first two patients also showed reduced profusion in frontal as well as temporal cortical regions (unlike those with more normal primacy effects). In the general discussion, we consider the view that prefrontal regions make a critical contribution to the recall of serial order.
} 
Scotti, \& Spinnler, 1972; Gainotti, Miceli, \& Caltagirone, 1979; Gainotti, Silveri, Villa, \& Caltagirone, 1983; Saygin, Dick, Wilson, Dronkers, \& Bates, 2003; Spinnler \& Vignolo, 1966). This pattern of deficits cannot be explained in terms of a disconnection between semantic and lexical representations.

An alternative view is that when multimodal comprehension problems occur in stroke aphasia (as opposed to difficulties confined to verbal tasks), this impairment reflects a breakdown in the executive processes that help to direct and shape linguistic and semantic activation in a task-appropriate fashion (Jefferies \& Lambon Ralph, 2006). The prefrontal cortex and temporoparietal junction are thought to form a single distributed system underpinning cognitive control (see Collette, Hogge, Salmon, \& Van der Linden, 2006; Peers et al., 2005). Damage to this cognitive control system would have profound consequences for performance on both semantic and verbal STM tasks. In semantic association tasks, for example, executive control is required to select the aspects of knowledge that are relevant to establishing a link between the probe and target. Similarly, in ISR, it is necessary to direct activation away from the most recently presented items and towards items that were presented earlier in the sequence, in order to recall items in serial order. Patients with SD, in contrast, are thought to have a gradually degrading store of semantic knowledge, in line with the hypothesis that the anterior temporal lobes act as a "convergence zone" for the formation of amodal semantic representations (see Rogers et al., 2004). This loss of semantic information in SD might disrupt the coherence of word forms in ISR (in line with the semantic binding hypothesis), but should not prevent activation from being appropriately directed towards the phonological segments that were presented at the beginning of the list.

Evidence for this distinction comes from a recent case-series comparison of patients with multimodal semantic problems following stroke aphasia and SD (Jefferies \& Lambon Ralph, 2006). The SD patients showed strong correlations between different semantic tasks - regardless of input/output modality - and substantial consistency when a set of items was assessed several times. They were also highly sensitive to frequency/ familiarity, made coordinate and superordinate semantic errors in picture naming and were insensitive to cueing, pointing to a graded loss of amodal semantic knowledge (see also Bozeat et al., 2000; Rogers et al., 2004). In contrast, patients with stroke aphasia (including the TSA cases in the current study) were inconsistent across tasks requiring different types of semantic processing: they were able to retrieve the information appropriate for one semantic test but were unable to reshape this information for another task, even when the same concept was being tapped. They were also insensitive to familiarity/frequency — instead, tests of semantic association were influenced by the ease with which relevant semantic relationships could be identified and distractors rejected. The stroke aphasic patients' comprehension impairment was exaggerated when small sets of semantically related items were presented rapidly and repeatedly, producing strong competition with the target response (Jefferies, Baker, Doran, \& Lambon Ralph, 2007). In addition, unlike SD patients, the aphasic patients made picture naming errors that were associatively rather than categorically related to the target (e.g., squirrel $\rightarrow$ "nuts"; glass $\rightarrow$ "ice"; lorry $\rightarrow$ "diesel"). These errors were apparently driven by strong but irrelevant semantic associations. The aphasic patients were highly responsive to phonemic cueing in picture naming presumably because the cues boosted activation of the target word relative to semantically related competitors (Jefferies, Corbett, Hopper, \& Lambon Ralph (submitted for publication-a)). All these features are consistent with a semantic control deficit: indeed, semantic difficulties were concomitant with executive impairment in the aphasic group (see also Baldo et al., 2005; Wiener, Connor, \& Obler, 2004). In contrast, nonverbal reasoning was largely intact in the SD group.

It is important to establish whether comprehension impairment in SD and stroke aphasia affects verbal STM in the same way because differences between the patient groups would motivate a major revision of the neuropsychologically inspired models described above (i.e. the inclusion of executive control processes). Yet ISR has never been directly compared in SD and stroke aphasia. The interpretation of the existing literature is also complicated by important methodological differences between the studies of SD and stroke aphasia. SD patients have intact phonology - i.e. they do well on tests requiring phonological judgements, do not make phonological errors in picture naming or spontaneous speech and have normal digit span (at least until the late stages of the disease; Jefferies et al., 2005). Consequently, it is appropriate to test their ISR using lists of four or five items. In contrast, aphasic participants often have a mixture of phonological and semantic deficits, much poorer ISR than is typical in SD and are asked to repeat very short lists - typically single items or pairs of items (see Martin \& Saffran, 1997). This difference alone might affect the impact of semantic impairment on verbal STM (see Forde \& Humphreys, 2002).

Although SD patients apparently always make frequent phoneme migration errors in ISR (Jefferies et al., 2004a), little is known about variability between comprehension-impaired stroke aphasic patients. Previous studies have typically only described one or two aphasic cases selected on the basis that they show interesting STM phenomena (e.g. Martin \& Lesch, 1996; Martin et al., 1999, 1994). The study by Martin and Saffran (1997) did report data from a larger group of aphasic patients but focussed on correlations between verbal 
STM and semantic/phonological impairment making it difficult to consider the extent of individual differences.

In summary, current theories of the role of lexical/ semantic knowledge in verbal STM would expect (a) similar effects of comprehension impairment on ISR in SD and stroke aphasia and (b) the predicted pattern of ISR impairment to be observed universally within each of these groups. There are indications from the current literature, however, that this may not be the case. Accordingly, the current study directly compared ISR in SD and TSA. If differences between these two patient groups are confirmed, then hypotheses such as the IA model (Martin \& Saffran, 1997) and the semantic binding theory (Patterson et al., 1994) will require modification. We opted to examine TSA cases (as opposed to semantically impaired stroke aphasic cases with poorer repetition, e.g. patients with Wernicke's aphasia) because the TSA patients had a language profile similar to that seen in SD: i.e. poor comprehension of both verbal and nonverbal stimuli in the context of fluent speech and good repetition (Albert, Goodglass, Helms, Rubens, \& Alexander, 1981; Berthier, 2000). This made it possible to test the two patient groups on exactly the same lists for the first time, circumventing the difficulties in interpretation mentioned above.

\section{Participants}

\section{Patients}

There were five SD patients recruited from St. Martin's Hospital, Bath, UK. At the time of testing, the patients fulfilled all of the published criteria for SD (e.g., Hodges et al., 1992): they had word-finding difficulties in the context of fluent speech and showed impaired semantic knowledge and single word comprehension; in contrast, phonology, syntax, visual-spatial abilities and day-to-day memory were relatively well preserved. MRI revealed focal atrophy of the inferior temporal lobes in every case (except KI for whom scanning was unavailable). Several years after this study was completed, BS developed a more global dementia that included marked amnesia for recent events. See Jefferies et al. (2005) for further details of these cases.

The SD patients were compared with five TSA patients recruited from stroke clubs and speech and language therapy services in Manchester, UK. Every case had chronic impairment from a CVA at least a year previously. The patients were selected on the basis that they had fluent speech, good word repetition and poor performance on both picture and word tests of semantic association (Camel and Cactus Test, described below). Therefore, their presentation was at least superficially similar to SD. Table 2 shows biographical/neuroimaging details and aphasia classifications from the Boston Diagnostic Aphasia Examination (Goodglass, 1983).

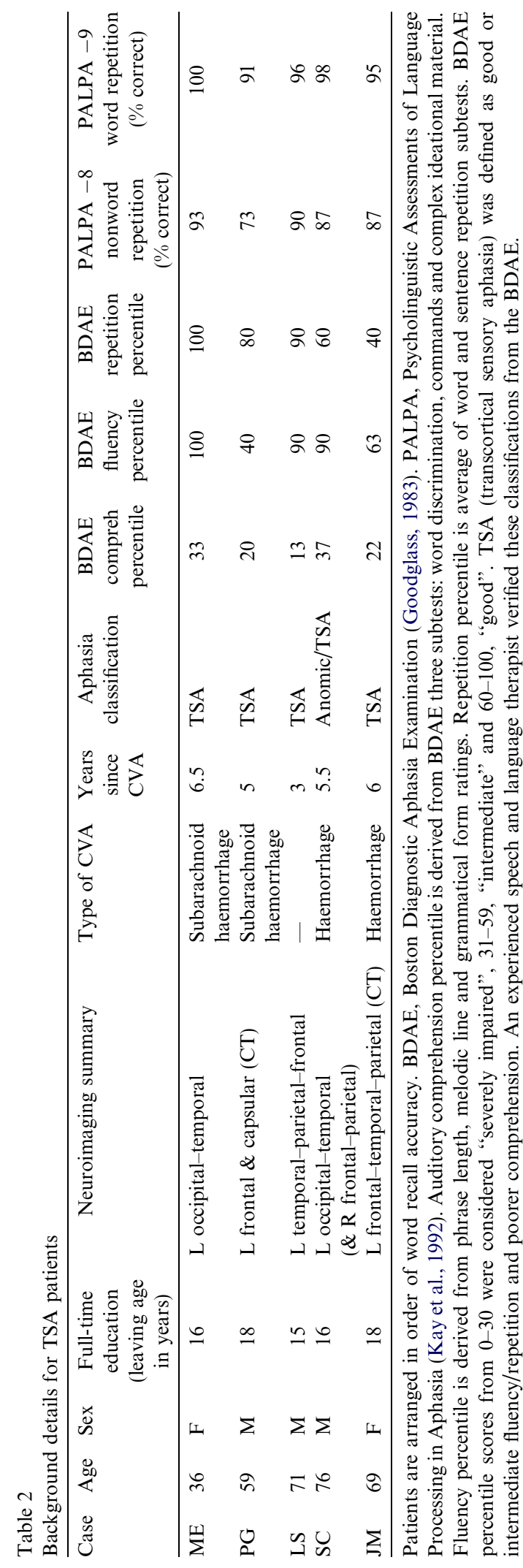


The patients in each group are arranged in order of word recall accuracy in the tables and graphs below.

\section{Control participants}

The patients were compared with two groups of healthy controls. (1) Jefferies et al. (2005) previously described the SD patients' performance on some of the tasks used here (recall of phonologically similar/dissimilar letters and words vs. nonwords). For these experiments, there were 10 or 12 controls matched to the SD group for age (late 50 s to early 70 s) and educational level (left full-time education aged 14-16). The TSA patients had a wider range of ages and educational levels (see Table 2). (2) The patients were also tested on an additional task (recall of words varying in frequency and imageability). For this experiment, data was collected from seven healthy controls aged 45-74 who left full-time education by the age of 18 .

\section{Background neuropsychological tests}

A range of measures were used to examine the patients' semantic and phonological abilities (see Table 3). Semantic abilities were assessed using four tests: the
Pyramids and Palm Trees (PPT) test of semantic association (Howard \& Patterson, 1992), in which items are presented as pictures and words; picture naming using 64 black-and-white line drawings from the Snodgrass and Vanderwart (1980) set; spoken word to picture matching using the same 64 items, each presented with nine semantically related distractors (Bozeat et al., 2000); and a synonym judgement test in which patients were asked to decide which of three written words was most similar in meaning to a target word. All of the SD patients performed below normal levels on each of these tests. The TSA patients were below the level of controls on all tests except for one patient (SC), who was in the normal range for PPT. Both sets of patients had similar levels of semantic impairment: the SD patients scored more poorly than the TSA patients on the word version of the PPT (Mann-Whitney $U=2.0$, $p<.05)$ but there were no significant differences on the other tests (all $p>.2)$. Further details about the TSA patients' comprehension impairment are available in Jefferies and Lambon Ralph (2006).

Four tests assessed phonological skills: minimal pairs discrimination for words and nonwords from the PALPA battery (Kay, Lesser, \& Coltheart, 1992); a phoneme segmentation task in which patients added or removed single phonemes from presented words/

Table 3

Neuropsychological assessments of SD and TSA patients

\begin{tabular}{|c|c|c|c|c|c|c|c|c|c|c|c|c|c|c|c|}
\hline \multirow[t]{2}{*}{ Test } & \multirow[t]{2}{*}{ Max } & \multicolumn{5}{|c|}{ TSA } & \multicolumn{5}{|c|}{ SD } & \multicolumn{2}{|c|}{ Mean } & \multicolumn{2}{|c|}{ Controls } \\
\hline & & ME & PG & LS & $\mathrm{SC}$ & $\mathrm{JM}$ & BS & KI & JT & EK & SJ & TSA & SD & Mean & Cut-off $^{b}$ \\
\hline \multicolumn{16}{|l|}{ Semantic tests } \\
\hline \multicolumn{16}{|l|}{ PPT } \\
\hline Pictures & 52 & $29^{\mathrm{a}}$ & $42^{\mathrm{a}}$ & $31^{\mathrm{a}}$ & 50 & $35^{\mathrm{a}}$ & $33^{\mathrm{a}}$ & $31^{\mathrm{a}}$ & $35^{\mathrm{a}}$ & $30^{\mathrm{a}}$ & $48^{\mathrm{a}}$ & 37 & 35 & 51 & 49 \\
\hline Words & 52 & $39^{\mathrm{a}}$ & $43^{\mathrm{a}}$ & $39^{\mathrm{a}}$ & 51 & $44^{\mathrm{a}}$ & $35^{\mathrm{a}}$ & $35^{\mathrm{a}}$ & $31^{\mathrm{a}}$ & $35^{\mathrm{a}}$ & $42^{\mathrm{a}}$ & 43 & 36 & 51 & 48 \\
\hline Naming & 64 & $4^{\mathrm{a}}$ & $44^{\mathrm{a}}$ & $5^{\mathrm{a}}$ & $28^{\mathrm{a}}$ & $30^{\mathrm{a}}$ & $29^{\mathrm{a}}$ & $15^{\mathrm{a}}$ & $6^{\mathrm{a}}$ & $18^{\mathrm{a}}$ & $30^{\mathrm{a}}$ & 22 & 20 & 62 & 59 \\
\hline Spoken word-picture matching & 64 & $50^{\mathrm{a}}$ & $58^{\mathrm{a}}$ & $37^{\mathrm{a}}$ & $59^{\mathrm{a}}$ & $53^{\mathrm{a}}$ & $40^{\mathrm{a}}$ & $36^{\mathrm{a}}$ & $34^{\mathrm{a}}$ & $39^{\mathrm{a}}$ & $59^{\mathrm{a}}$ & 51 & 42 & 64 & 63 \\
\hline Synonym judgement & 96 & $81^{\mathrm{a}}$ & $69^{\mathrm{a}}$ & $47^{\mathrm{a}}$ & $71^{\mathrm{a}}$ & $27^{\mathrm{a}}$ & NT & $58^{\mathrm{a}}$ & NT & $68^{\mathrm{a}}$ & $78^{\mathrm{a}}$ & 59 & 68 & 95 & 91 \\
\hline \multicolumn{16}{|l|}{ Phonological tests } \\
\hline \multicolumn{16}{|l|}{ Minimal pairs } \\
\hline Words & 72 & 71 & 69 & 67 & 63 & 65 & 71 & 69 & 70 & 71 & 72 & 67 & 71 & 70 & 63 \\
\hline Nonwords & 72 & 65 & $59^{\mathrm{a}}$ & $61^{\mathrm{a}}$ & $60^{\mathrm{a}}$ & $46^{\mathrm{a}}$ & 71 & 71 & 70 & 71 & 70 & 58 & 71 & 71 & 65 \\
\hline \multicolumn{16}{|l|}{ Phoneme segmentation } \\
\hline Addition & 48 & 37 & $15^{\mathrm{a}}$ & $0^{\mathrm{a}}$ & TA & $20^{\mathrm{a}}$ & 48 & $38^{\mathrm{a}}$ & 44 & $37^{\mathrm{a}}$ & 45 & 18 & 42 & $\mathrm{c}$ & $\mathrm{c}$ \\
\hline Deletion & 48 & $39^{\mathrm{a}}$ & $29^{\mathrm{a}}$ & $0^{\mathrm{a}}$ & $29^{\mathrm{a}}$ & $10^{\mathrm{a}}$ & 48 & 45 & 46 & 44 & 47 & 21 & 46 & $\mathrm{c}$ & $\mathrm{c}$ \\
\hline Rhyme judgement & 48 & $46^{\mathrm{a}}$ & $39^{\mathrm{a}}$ & $25^{\mathrm{a}}$ & $29^{\mathrm{a}}$ & $31^{\mathrm{a}}$ & $46^{\mathrm{a}}$ & $40^{\mathrm{a}}$ & $46^{\mathrm{a}}$ & $42^{\mathrm{a}}$ & $43^{\mathrm{a}}$ & 34 & 43 & 48 & 47 \\
\hline Rhyme production $^{\mathrm{d}}$ & 24 & 19 & $12^{\mathrm{a}}$ & $8^{\mathrm{a}}$ & TA & $0^{\mathrm{a}}$ & 24 & 23 & 20 & 23 & 22 & 10 & 23 & 22 & 17 \\
\hline \multicolumn{16}{|l|}{ Digit span } \\
\hline Forwards & - & 6 & 6 & $4^{\mathrm{a}}$ & 6 & $3^{\mathrm{a}}$ & 8 & 8 & 8 & 7 & 5 & 5.0 & 7.2 & 7 & 5 \\
\hline Backwards & - & 3 & $2^{\mathrm{a}}$ & $1^{\mathrm{a}}$ & $2^{\mathrm{a}}$ & $2^{\mathrm{a}}$ & 4 & 5 & 4 & 4 & 3 & 2.0 & 4.0 & 5 & 3 \\
\hline
\end{tabular}

Patients are arranged in order of word recall accuracy.

${ }^{\text {a }}$ Indicates abnormal performance. SD, semantic dementia. TSA, transcortical sensory aphasia. PPT, pyramids and palm trees. NT, not tested. TA, testing abandoned.

${ }^{\mathrm{b}}$ Cut-off for normal participants at two SD below mean.

c Controls perform at ceiling on these tests (Bird et al., 2003).

${ }^{\mathrm{d}}$ Includes rhyming nonword responses. 
nonwords to produce new items (Patterson \& Marcel, 1992); a rhyme judgement task which required patients to decide whether two auditorily presented words rhymed (Patterson \& Marcel, 1992); and a rhyme production task in which patients attempted to produce a word that rhymed with a spoken word (Patterson \& Marcel, 1992). These data have been reported elsewhere for the SD patients (Jefferies et al., 2005). All of the SD patients performed normally on the minimal pairs task but there was evidence of some mild impairment on the remaining three tests. This might have reflected the impact of their semantic impairment on phonological integrity (Jefferies et al., 2005).

The TSA patients all showed some evidence of phonological weakness: one patient (LS) failed to produce any correct responses during phoneme segmentation and testing on this task was abandoned for another (SC). Testing was also abandoned for SC on the rhyme production task. On the remaining two phonological tests, performance was better but still below the normal range for all patients except ME. However, these impairments might have reflected the underlying deficit of cognitive control seen in TSA, rather than an inability to deal with the phonological demands of the tasks (Jefferies, Crisp, \& Lambon Ralph, 2006). Evidence for this comes from the patients' errors on the production tasks. The two most impaired patients (LS, JM) frequently responded with words that were semantically related to the presented word (e.g., white $\rightarrow$ "brown"). Two other patients (SC, PG) often perseverated on words produced earlier in the test.

SD patients outperformed TSA patients on nonword minimal pair discrimination (Mann-Whitney $U=0$, $p<.01)$. The group difference approached significance for real word minimal pairs (Mann-Whitney $U=3.5$, $p=.056$ ) and rhyme judgment (Mann-Whitney $U=$ $4.0, p=.095)$. There is evidence therefore that the TSA group performed more poorly on phonological tests than the SD patients, but neither group had substantial phonological deficits.

The forward and backward digit spans of the patients were also assessed using the Wechsler Memory Scale (Wechsler, 1987). The SD patients all had normal spans, measured both backwards and forwards (see Table 3). Two of the five TSA patients had reduced forward digit span (JM, LS) and all had difficulty when repeating digits backwards. However, digit span for our TSA patients was less impaired than for Martin and Saffran's (1997) cases, making it possible to test the TSA and SD groups on the same list lengths.

\section{Phonological similarity}

Normal ISR is poorer for similar sounding items compared with phonologically more distinct items. This 'phonological similarity effect' is usually taken as evidence for phonological coding in verbal STM (Baddeley, 1966; Conrad, 1964; Conrad \& Hull, 1964) and is reduced/eliminated in patients with impaired retention of phonological information (Martin et al., 1994). Given that patients with semantic impairment following SD or stroke aphasia are expected to rely on phonological information in STM, effects of phonological similarity in ISR are predicted for both groups.

\section{Methods}

The patients and 12 controls recalled lists of phonologically similar and dissimilar letters in serial order. The phonologically similar set contained the letters E, C, T, $\mathrm{P}, \mathrm{V}, \mathrm{B}, \mathrm{G}, \mathrm{D}$ and the dissimilar set consisted of the letters W, S, Q, Y, R, J, F, L, following Knott et al. (2000). There were forty lists; half contained four items and half contained six items. The phonologically similar and dissimilar letters were blocked using an ABBA design. Items were read aloud at a rate of one item per second for spoken recall. Two TSA patients, JM and PG, were not tested on the longer list length.

\section{Results}

\section{Accuracy}

Fig. 1 shows the results for this task. The groups were compared using a within-subjects ANOVA, combining data across the two list lengths. The main effects of phonological similarity $(F(1,19)=59.6, \quad p<.0001)$ and group $(F(2,19)=16.0, \quad p<.0001)$ were significant. Recall accuracy was higher for the SD vs. TSA patients $(t(8)=3.2,95 \% \mathrm{CI}=6.6-41.9 ; p=.01$, planned comparison) and did not differ for the SD patients vs. normal controls $(t(15)<1)$. There was no difference in the

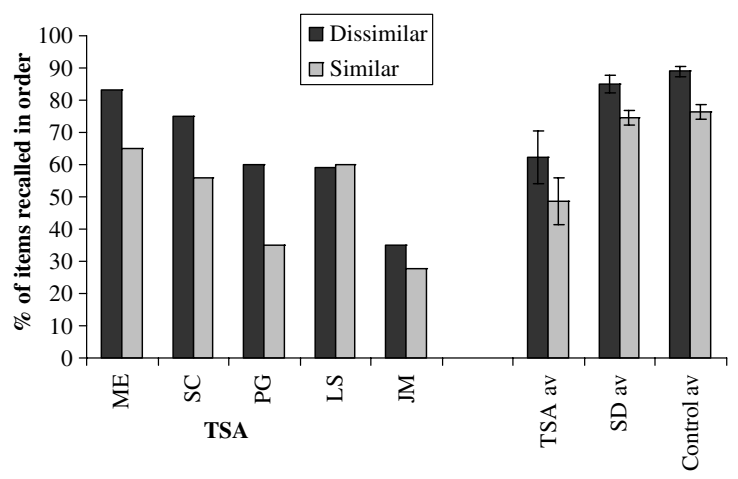

Fig. 1. Effect of phonological similarity on ISR for letters for individual TSA patients. Figure combines data across list lengths. The patients are arranged in order of word recall accuracy. Error bars indicate one standard error of mean. Data for individual SD patients is available in Jefferies et al., 2005. 
size of the phonological similarity effect between the groups $(F(2,19)<1)$. Three of the SD patients (SJ, BS, EK) showed a significant recall advantage for phonologically dissimilar letters but two others (KI, JT) did not (see Jefferies et al., 2005). Similarly, three TSA patients (SC, PG, ME) showed a significant recall advantage for phonologically dissimilar letters (Fisher's exact test: one-tailed $p<.02)$ and two did not (JM, LS). The minimum ISR difference between phonologically dissimilar and similar items was $6 \%$ in the controls: one CVA patient (LS) and one SD patient (JT) fell outside this range.

\section{Errors}

The poorer recall of the TSA group was largely accounted for by an increase in omissions. While there were no marked differences between the groups in the proportions of order and item errors (i.e. items recalled in the wrong serial position vs. not recalled at all), there were some qualitative differences in the errors seen. Several patients in the TSA group (particularly SC and JM) repeatedly produced familiar letter strings such as "BBC"; this type of response was never observed in the SD group. One TSA case, LS, also showed frequent perseverations of letters from earlier lists.

\section{Discussion}

The SD and TSA groups showed effects of phonological similarity in ISR that did not differ significantly from control performance, suggesting that their recall relied on phonological maintenance in a relatively normal way. Although not every individual case showed significantly poorer recall of phonologically similar letters, the size of this effect was variable for the controls. The frequent perseverations and "letter completion" errors (e.g., BBC) for JM and LS in the TSA group may also have masked any potential effect of phonological similarity.

\section{Lexicality}

Previous studies have suggested that both SD and stroke aphasic patients with semantic STM impairments show reduced effects of lexicality in ISR (e.g., Jefferies et al., 2005; Martin et al., 1994). We established if this was equally true of our TSA and SD patients by comparing their word/nonword recall with that of controls.

\section{Methods}

The patients and 10 control participants were asked to repeat lists of monosyllabic words and nonwords (taken from Gathercole, Pickering, Hall, \& Peaker, 2001). The patients were presented with two to four items and the controls were additionally tested on fiveitem lists. At each length, there were twenty lists in each condition. The words and nonwords, which were not repeated in the course of the experiment, were blocked using an ABBA design and were presented auditorily at a rate of one item per second for ISR.

\section{Results}

\section{Accuracy}

In order to compare the size of the lexicality effect across the three groups, we combined data from lengths $2-4$ for the patients and 3-5 for the controls; these results are shown in Fig. 2. Both patient groups showed weaker than normal lexicality effects: there was a significant lexicality by group interaction when the SD patients were compared with controls $(F(1,13)=46.7, p<.0001)$ and for the TSA patients vs. controls $(F(1,13)=14.5, p=.002)$. The lexicality effect was also smaller for SD vs. TSA patients $(F(1,8)=5.8, p=.04)$. As noted by Jefferies et al. (2005), the SD patients' recall was substantially impaired for words but was normal for nonwords; this was true of all five of the patients in the current sample (individual SD patient data is available in Jefferies et al., 2005). In contrast, four of the five TSA patients showed impaired recall of both words and nonwords (ME was within the normal range for both materials; see Fig. 2) and all five TSA patients showed a significant recall advantage for the words (Fisher's exact one-tailed $p<.002$ ).

\section{Errors}

Errors were examined by combining lengths $2-4$ for the patients and 3-5 for the controls. The majority of errors were phonological for every group, accounting

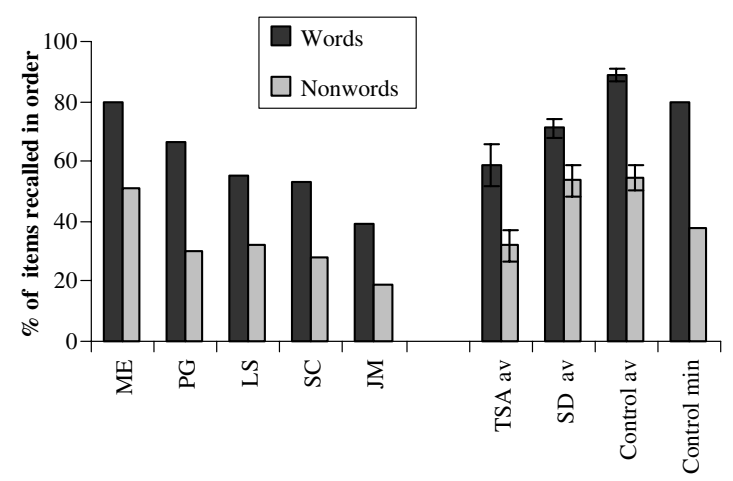

Fig. 2. Effect of lexicality for individual TSA patients. Figure combines data from list lengths 2-4. The TSA patients are arranged in order of word recall. Error bars indicate one standard error of mean. Control min, minimum performance in each condition. 
for $48 \%, 76 \%$ and $44 \%$ of errors in word recall and $59 \%$, $79 \%$ and $65 \%$ of errors in nonword recall for the TSA, $\mathrm{SD}$ and control groups, respectively. There were also frequent omissions and "unrelated" errors which preserved fewer than half of the target phonemes. Order errors were rare, accounting for less than $3 \%$ of the errors in all three groups. In nonword ISR, around half of the errors were words for all three groups (combining the phonological and unrelated error categories). In contrast, the controls showed a strong tendency to recall words as real words (lexicality was preserved in $87 \%$ of errors). This effect was weaker for the TSA cases $(76 \%$ of errors were words) and weaker still for the SD patients $(61 \%$ words). In the word recall task, a smaller proportion of errors were words for both patient groups relative to controls (Fisher's exact two-tailed $p<.03$ ). The SD patients also showed a weaker lexical bias than the TSA cases $(p<.02)$.

\section{Discussion}

The TSA and SD patients both showed smaller lexicality effects than the controls. This was more marked for the SD group, perhaps because the TSA patients had some additional phonological problems that impaired nonword recall.

\section{Frequency and imageability}

SD patients show effects of word frequency/imageability in ISR (Knott et al., 1997, 2000; Majerus et al., 2007; McCarthy \& Warrington, 2001). These effects might be larger than those seen in normal recall because the degradation of concepts in SD is strongly related to frequency (Bozeat et al., 2000; Funnell, 1995). Accordingly, semantic memory may make a relatively normal contribution to the recall of high frequency words but provide little support for uncommon words (Jefferies et al., 2006). Similarly, highly imageable words may be more robust to semantic degradation than abstract items due to their richer semantic representations, resulting in large imageability effects in ISR through the same mechanism (Jones, 1985; Plaut \& Shallice, 1991). In contrast, semantic impairment in stroke aphasia is thought to reduce the influence of these variables (Martin \& Saffran, 1997), although some TSA cases have shown better recall of high than low frequency words (Berthier, 2001; Martin \& Saffran, 1990). However, little is known about the size of frequency/imageability effects in these patient groups relative to normal controls. We directly compared the impact of these lexical-semantic variables in our two groups of patients and healthy volunteers. ${ }^{2}$

\footnotetext{
${ }^{2}$ JM dropped out of testing at this stage in the study and did not take part in any further experiments.
}

\section{Methods}

This experiment was used to examine (1) the effects of frequency/imageability on ISR; (2) errors at the level of whole items, including the tendency to repeat items out of serial order; (3) phoneme migration errors; and (4) serial position effects (see separate sections below). The patients and seven controls repeated forty lists of five monosyllabic words. The TSA patients were also tested on three-item lists (constructed by deleting two middle items from the longer lists). Frequency was crossed with imageability, with two levels of each variable, and the all of the words in a single list were taken from a single frequency by imageability condition. The characteristics of the words are given in Appendix A. All words had a CVC structure. Lists were presented auditorily at a rate of one word per second in a random order. If participants failed to give the correct number of responses, we positioned omissions in a way that minimised the number of phonological errors in the response. For example, if a participant responded to the list "kale, zone, rung, dip, verb" with "phone, rail, dip", it was assumed that the first and last words in the list had been omitted from the response. Participants were not asked to say "pass" when they omitted an item because this might have interfered with the tendency of phonemes to migrate. This method has been used previously in studies of phoneme migrations in normal ISR (Jefferies et al., 2006).

\section{Results}

\section{Accuracy}

In the analyses presented below, we scored responses as correct if they were produced in the appropriate serial order relative to other items (regardless of exact serial position). That is, an item was judged to be incorrect if it was produced before an item that preceded it in the original list. This scoring method minimised the effect of intrusions and attempts at self-correction on the number of item order errors and therefore yielded a clearer difference between the groups.

The effect of frequency and imageability on recall accuracy is shown in Fig. 3. The five-item lists were examined using ANOVA including frequency, imageability and participant group as factors. The analysis was carried out using participants as cases $\left(F_{1}\right)$ and treating each list as a separate case $\left(F_{2}\right)$. Both analyses revealed significant effects of patient group $\left(F_{1}(2,13)=31.5, \quad p<.001\right.$; $\left.F_{2}(2,72)=278, p<.001\right)$. Bonferroni post-hoc tests indicated that the controls performed better than the SD patients $\left(t_{1}(10)=5.45,95 \% \mathrm{CI}=22.2-52.8, p<.001\right.$; $\left.t_{2}(39)=15.3,95 \% \mathrm{CI}=33.2-43.4, p<.001\right)$, as well as the TSA cases $\left(t_{1}(9)=9.09,95 \% \mathrm{CI}=36.2-60.1, p<\right.$ $\left..001 ; t_{2}(39)=24.5,95 \% \mathrm{CI}=46.7-55.1, p<.001\right)$. The 

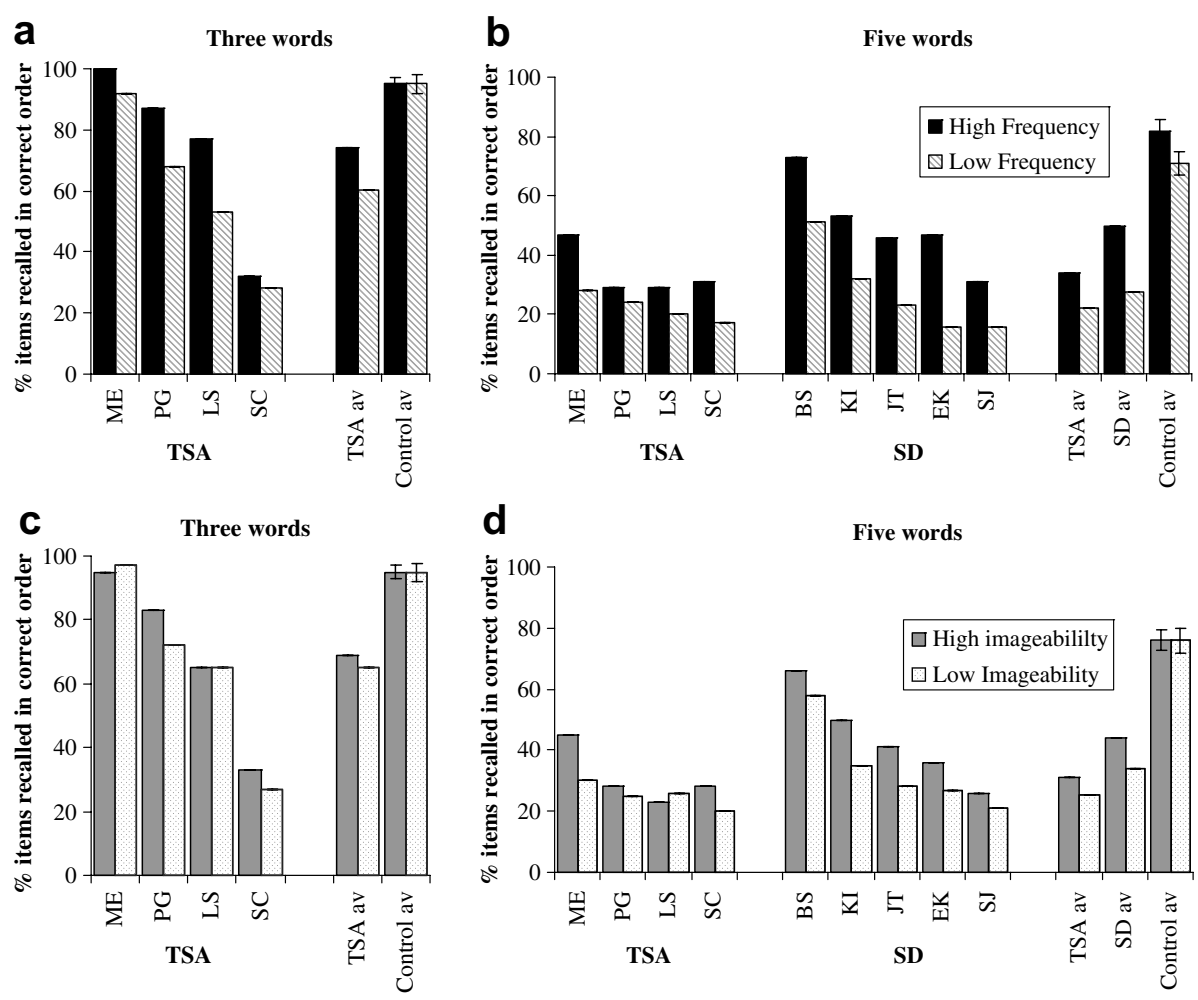

Fig. 3. Recall of three and five-word lists (Figs. 3(a) and 3(b) show effects of word frequency and Figs. 3(c) and 3(d) show effects of imageability for three- and five-item lists respectively). Patients are arranged in order of overall word recall. Error bars indicate one standard error of mean.

TSA patients also performed more poorly than the SD patients, though the difference only reached significance in one analysis $\left(t_{1}(7)=1.35\right.$, n.s.; $t_{2}(39)=4.94,95 \%$ $\mathrm{CI}=7.4-17.7, p<.001)$.

ISR was more accurate for both high vs. low frequency words $\left(F_{1}(1,13)=85.3, p<.001 ; F_{2}(1,36)=70.0, p<\right.$ $.001)$ and high vs. low imageability words $\left(F_{1}(1,13)=\right.$ $\left.10.0, p<.01 ; F_{2}(1,36)=8.55, p<.01\right)$. There was also a interaction between frequency and group $\left(F_{1}(2,13)=\right.$ $\left.5.42, p<.02 ; F_{2}(2,72)=4.47, p<.02\right)$. The effect of frequency was greater in the SD cases than in controls $\left(F_{1}(1,10)=9.48, p=.012 ; F_{2}(1,36)=6.81, p=.013\right)$. In addition, the SD patients showed larger effects of frequency than the TSA cases $\left(F_{1}(1,7)=7.29, p<.03\right.$; $\left.F_{2}(1,36)=5.27, p<.03\right)$. Frequency effects in TSA did not differ from those in controls (both $F<1$ ), though it should be noted that significant frequency effects were observed in both groups (TSA: $t_{1}(3)=3.87,95 \%$ $\mathrm{CI}=2.1-21.4, \quad p<.05 ; \quad t_{2}(39)=4.58, \quad 95 \% \quad \mathrm{CI}=6.6-$ 16.9, $p<.001$; Controls: $t_{1}(6)=4.21,95 \% \mathrm{CI}=4.6-$ $17.2, \quad p<.02 ; \quad t_{2}(39)=4.70, \quad 95 \% \quad \mathrm{CI}=6.2-15.6$, $p<.001)$. Additionally, the interaction between imageability and group approached significance $\left(F_{1}(2,13)=\right.$ $\left.3.58, p=.06 ; \quad F_{2}(2,72)=2.74, p=.07\right)$. SD patients showed a larger advantage for high imageability words than controls $\left(F_{1}(1,10)=7.93, p=.02 ; F_{2}(1,36)=5.15\right.$, $p=.03)$. However, the size of the imageability effect in TSA did not differ from the other two groups (all $F<2.3, p>.1)$.

The TSA patients showed similar results on shorter lists containing only three words (SD patients were not tested on the shorter lists and controls were at ceiling: mean recall accuracy was $95 \%$ ). The TSA patients showed an effect of frequency $\left(t_{1}(3)=2.99,95 \% \mathrm{CI}=-.8-27.6\right.$, $\left.p=.06 ; t_{2}(38)=3.54,95 \% \mathrm{CI}=5.6-20.8, p<.001\right)$ but no effect of imageability $\left(t_{1}(3)=1.37\right.$, n.s.; $t_{2}(38)=1.01$, n.s.).

Turning to results for individual cases, all of the patients were impaired on the task with the exception of one SD patient, BS, who performed within the normal range. All of the SD patients showed a significant advantage for high over low frequency words (Fisher's exact one-tailed $p<.01$ ), but only two (JT, KI) performed better on high compared with low imageability words $(p<.04)$. Two TSA patients (ME, SC) recalled high frequency words more accurately than low frequency words (Fisher's exact one-tailed $p<.02$ ) and one (ME) showed a significant effect of imageability $(p<.02)$. 
Table 4

Errors made by each patient in ISR

\begin{tabular}{|c|c|c|c|c|c|c|c|c|c|c|c|c|c|}
\hline & \multicolumn{4}{|c|}{ TSA } & \multicolumn{5}{|c|}{ SD } & \multirow{2}{*}{$\begin{array}{l}\text { TSA } \\
\text { Mean }\end{array}$} & \multirow{2}{*}{$\begin{array}{c}\text { SD } \\
\text { Mean }\end{array}$} & \multicolumn{2}{|c|}{ Controls } \\
\hline & ME & PG & LS & $\mathrm{SC}$ & BS & KI & JT & EK & SJ & & & Mean & $S D$ \\
\hline \multicolumn{14}{|l|}{ Three-word lists } \\
\hline Omissions & 0 & .01 & 0 & $.48^{\mathrm{a}}$ & \multirow{3}{*}{\multicolumn{5}{|c|}{ Not tested }} & .12 & & $<.01$ & $<.01$ \\
\hline Intrusions & .04 & $.21^{\mathrm{a}}$ & $.35^{\mathrm{a}}$ & $.23^{\mathrm{a}}$ & & & & & & .21 & & .05 & .06 \\
\hline Order errors & 0 & .01 & 0 & 0 & & & & & & $<.01$ & & 0 & 0 \\
\hline \multicolumn{14}{|l|}{ Five-word lists } \\
\hline Omissions & .19 & $.52^{\mathrm{a}}$ & .16 & $.64^{\mathrm{a}}$ & .16 & .02 & .01 & .04 & .05 & .38 & .06 & .07 & .07 \\
\hline Intrusions & $.39^{\mathrm{a}}$ & .14 & $.44^{\mathrm{a}}$ & .13 & .20 & $.55^{\mathrm{a}}$ & $.64^{\mathrm{a}}$ & $.64^{\mathrm{a}}$ & $.71^{\mathrm{a}}$ & .27 & .55 & .14 & .07 \\
\hline Order errors & $.12^{\mathrm{a}}$ & $.22^{\mathrm{a}}$ & $.38^{\mathrm{a}}$ & .00 & .02 & .03 & .01 & .03 & .04 & .18 & .03 & .03 & .02 \\
\hline
\end{tabular}

Patients are arranged in order of word recall accuracy. $S D$, standard deviation. Order errors are reported as a proportion of words correctly recalled in any order. Omission and intrusion errors are expressed as a proportion of items presented.

${ }^{a}$ Differs significantly from control performance (modified $t$-test, $p<.05$; Crawford and Garthwaite, 2002).

\section{Errors}

Omissions, intrusion errors and order errors are shown in Table 4. Intrusions include nonword responses as well as real words that were not present in the target list. Order errors are given as a proportion of the total words correctly produced in any order, as the probability of an item being recalled out of sequence increased as more words were recalled.

Poor performance in the SD group was attributable to a high rate of item intrusions; these responses were usually phonologically related to the target and in many cases represented the "phoneme migration" effect characteristic of recall in this patient group (see following section). The TSA patients also tended to make phonological errors on the three-word lists, and two patients (ME, LS) continued to make these errors on the longer lists. However, on five-item lists, omissions and order errors became more prominent features of recall in TSA. Whole items were produced out of sequence above control levels by ME, LS and PG (although not by SC because he frequently omitted items). As a group, the TSA patients were more likely than the SD cases to omit words $\quad\left(t_{1}(7)=2.96, \quad 95 \% \quad \mathrm{CI}=-.02-.32, \quad p=.06\right.$; $\left.t_{2}(39)=23.5,95 \% \mathrm{CI}=.30-.36, p<.001\right)$ and to recall words out of serial order $\left(t_{1}(7)=2.13,95 \% \mathrm{CI}=.07\right.$ $\left..59, p=.07 ; t_{2}(39)=7.05,95 \% \mathrm{CI}=.12-.22, p<.001\right)$. Conversely, the SD patients made more intrusion errors than the TSA patients on the five-item lists $\left(t_{1}(7)=2.19\right.$, $95 \% \quad \mathrm{CI}=-.02-.58, \quad p=.06 ; \quad t_{2}(39)=9.56, \quad 95 \%$ $\mathrm{CI}=.22-.34, p<.001)$.

The TSA patients' order errors followed a particular pattern: the last word in the target list was often produced at the beginning of the response. This error was particularly prominent for LS (affecting 90\% of trials) and PG (48\% of trials). In addition, these two patients recalled the fourth word from the list second on $33 \%$ and $23 \%$ of trials, respectively. Even on trials where terminal items were not recalled accurately, the responses made first and second by LS and PG were often phonemic paraphasias of the fifth and fourth target items. To some extent, SC also showed a similar pattern: he did not make order errors perhaps because of his frequent omissions but he still had better recall of the final list items and he produced these first on $10 \%$ of trials (see below). As a group, the final word presented was recalled first by TSA cases on $37 \%$ of trials. SD patients and controls did not show these tendencies.

\section{Discussion}

The SD patients showed larger frequency and imageability effects than controls in ISR. Frequency also had a larger effect in SD than in TSA. The TSA patients did show some effect of frequency but no advantage for highly imageable items, mirroring the pattern for controls. $^{3}$

The two groups also made different types of errors. The TSA patients often omitted words and showed a marked tendency toward repeating words out of serial order - in particular, the final list items were frequently produced first. This pattern has been observed previously for semantically impaired stroke aphasics and has been taken as evidence of pathologically rapid decay of lexical or semantic representations (Martin \& Ayala, 2004; Martin \& Lesch, 1996; Martin \& Saffran, 1990). Conversely, the errors made by the SD cases usually involved the production of phonologically related words or nonwords. This suggests disruption to the representations of individual words, rather than the processes that sequence whole words within a list.

\footnotetext{
${ }^{3}$ We cannot conclude that our TSA cases showed weaker than normal imageability effects (in line with the findings of Martin \& Saffran, 1997) as neither the TSA patients nor the controls were significantly affected by this variable.
} 


\section{Phoneme migrations}

Previous research has demonstrated that SD patients frequently make phoneme migrations or 'blend' errors in ISR, in which the constituent phonemes of a list are recombined to form new words or nonwords in recall (e.g., Patterson et al., 1994). This behaviour suggests that degradation of semantic knowledge results in a loss of phonological coherence in STM. TSA patients with poor semantic control might not show this pattern if their semantic representations are largely intact. Although phoneme migrations have been reported in one previous case of TSA (Martin \& Saffran, 1990), this is the first direct comparison of the prevalence of these errors in SD and TSA.

\section{Methods}

The responses made in the five-word ISR task were analysed at the level of individual phonemes. Phonemes were not repeated within each presented list, so we were able to trace all phoneme migration errors. A reported phoneme was classed as a phoneme migration error if it was produced in a different serial position to the one it occupied in the original list. Usually, this phoneme movement resulted in a new combination of phonemes from different words to form an intrusion error (e.g. cat, $\operatorname{dog} \rightarrow$ bat, $\operatorname{cog}$ ). ${ }^{4}$ However, there were some cases in which an entire word moved to a different position in the list (e.g. cat, $\operatorname{dog} \rightarrow \operatorname{dog}$, cat). The phonemes in these words were not considered to have migrated, as the phonology of the words remained intact.

\section{Results}

Rates of phoneme migrations for each patient are given in Fig. 4. When the three groups were compared, there was a significant group difference $\left(F_{1}(2,13)=9.14\right.$, $\left.p<.005 ; F_{2}(2,72)=70.2, p<.001\right)$. Bonferroni post-hoc tests confirmed that both patient groups made more phoneme migrations than controls (SD vs. control: $t_{1}(10)=$ $4.20,95 \% \mathrm{CI}=10.3-33.5, p<.01 ; t_{2}(39)=12.4,95 \%$ $\mathrm{CI}=18.4-25.6, p<.001$; TSA vs. control: $t_{1}(9)=3.58$, $95 \% \quad \mathrm{CI}=3.6-13.0, \quad p<.05 ; \quad t_{2}(39)=10.1, \quad 95 \%$ $\mathrm{CI}=12.2-18.3, p<.001)$. The migration rate was also higher in SD compared to TSA when examined by items $\left(t_{1}(7)=1.12, \quad\right.$ n.s.; $\quad t_{2}(39)=2.85, \quad 95 \% \quad \mathrm{CI}=2.0-11.6$, $p<.05)$. This effect may not have reached significance

\footnotetext{
${ }^{4}$ This analysis examined the source of phonemes that were produced by the patients. Phonemes that moved to the wrong serial position and recombined with the elements of other list items were treated as migrations even if the phoneme was also produced in the correct serial position (e.g., cat, dog $\rightarrow$ cat, $\operatorname{cog})$.
}
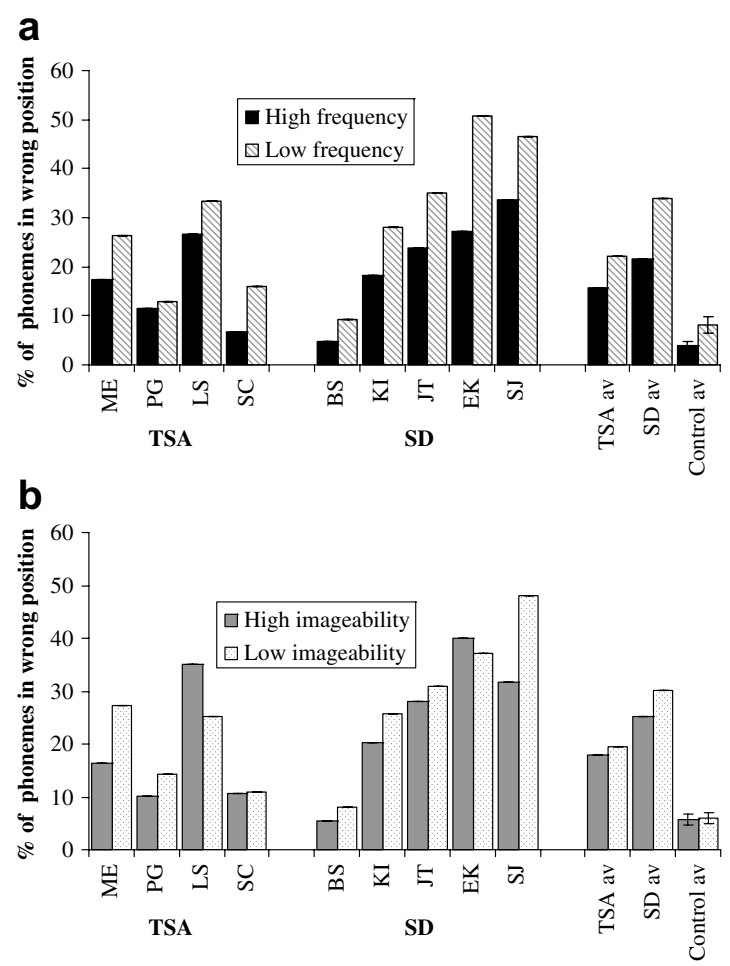

Fig. 4. Rate of phoneme migrations in five-word list recall. Fig. 4a shows effects of word frequency and Fig. $4 \mathrm{~b}$ shows effects of imageability. Error bars indicate one standard error of mean.

by subjects because of the small number of patients in each group.

Phoneme migrations occurred more often on low than high frequency lists $\left(F_{1}(1,13)=37.7, p<.001\right.$; $\left.F_{2}(1,36)=29.9, p<.001\right)$. Frequency had a bigger effect on the rate of phoneme migrations for the SD patients than the controls $\left(F_{1}(1,10)=6.77, p<.05 ; F_{2}(1,36)=\right.$ $6.72, p<.02)$, though neither group differed from the TSA cases (all $F<2.1, p>.2$ ). Imageability did not affect the overall rate of phoneme migrations $\left(F_{1}(1\right.$, $\left.13)=2.24, p>.1 ; F_{2}(1,36)=1.64, p>.2\right)$ and this variable did not interact with group (both $F<1$ ).

\section{Discussion}

For the SD patients, phonemes from different words frequently recombined in ISR to form incorrect words and neologisms. This finding is predicted by the "semantic binding" hypothesis, which states that the phonological coherence of words is undermined by semantic degradation (Patterson et al., 1994). Although the TSA patients also made a number of these errors, they were less common than in SD. As seen in the previous section, far more of the TSA patients' errors involved misordering words or failing to respond at all. Phoneme migrations were also more likely to occur for low frequency 
words, particularly in the SD group. This reinforces the notion that the semantic representations of low frequency words provide weaker constraints on phonology, particularly in SD where low frequency concepts are susceptible to degradation (Bozeat et al., 2000).

\section{Serial position effects}

Previous studies have suggested that SD and semantically impaired stroke aphasic patients show rather different serial position curves in ISR. SD cases typically show large primacy effects for both known and degraded words (Jefferies, Jones et al., 2004; Knott et al., 1997). Semantic deficits in stroke aphasia, on the other hand, are associated with robust recency effects and a reduction in primacy (Martin, Ayala, \& Saffran, 2002; Martin \& Saffran, 1997). However, due to the lack of direct comparisons between the two groups, methodological differences may account for these contrasting findings. Here, we examined serial position effects for the SD and TSA cases using the same three-, four- and five-word lists.

\section{Methods}

Serial position effects were examined using the word recall data presented above. The impact of increasing list length was examined for the SD and TSA groups using the three- and four-item lists from the lexicality experiment and the five-item lists from the frequency by imageability experiment, as both groups completed these experiments. The effect of list length on individual TSA patients was also examined using lists exclusively from the frequency by imageability experiment. This ensured that differences between three/four and five-item lists did not result from the use of different items at these list lengths in the previous analysis. The SD patients were not tested on threeitem lists in the frequency by imageability experiment, but unlike the TSA patients, they did not show any changes in the serial position curve with list length. To simplify comparisons across list lengths, scores from the middle serial positions were collapsed for lists of four and five items in statistical analyses. In order to give an accurate representation of the TSA patients' recall, items were scored as correct regardless of the order they were produced.

\section{Results}

Serial position curves for each group are shown in Fig. 5. Accuracy on initial and final words was compared with middle words using $\chi^{2}$ tests; these results are provided in Table 5. The SD patients and controls showed a significant primacy effect on lists of four and five items but the recency effect was not significant for either group. The TSA group displayed a flat serial position curve on four-item lists, but exhibited a sizeable recency effect for the five-item lists. This difference in serial position curves on the five item lists was supported by a 3 (patient group) $\times 5$ (serial position) ANOVA: the effect of serial position differed significantly between SD and TSA $(F(4,28)=7.30, p<.001)$ and between controls and TSA $(F(4,36)=6.78, p<.001)$, but there was no difference in the effect between SD patients and controls $(F<1)$.

The recency effect in the TSA group became much smaller if items were only counted as correct when recalled in serial order (see Fig. 5d), suggesting that the TSA patients' tendency to recall the final list items first gave rise to this effect. In contrast, the serial position curves for the SD patients and controls were relatively unaffected by this methodological modification.

Fig. 6 shows the serial position data for each TSA patient on the frequency by imageability lists containing three and five words. LS and PG in particular showed better recall of the initial list items on shorter lists but recency effects for the longer lists. On these longer lists, their ability to recall the items in serial order broke down and they showed a strong tendency to produce the terminal list item first.

\section{Discussion}

The serial position curves of the two groups of patients were similar for three- and four-item lists, but diverged when lists of five words were considered. The curves of the SD cases were parallel to those of controls at all list lengths: they showed a marked primacy effect on the longer lists but no advantage for recent items. Conversely, the TSA patients showed a large recency effect in the recall of five-item lists, with no primacy effect. These findings are consistent with previous studies that found robust primacy effects in SD and a reduction in primacy effects following semantic impairment in stroke aphasia. However, the recency effect in our TSA group was especially strong when the order in which items were produced was disregarded. This suggests that the advantage for recent items resulted from the patients' tendency to initiate recall with the terminal list item.

\section{General discussion}

This study directly compared the effect of semantic impairment on verbal STM in patients with semantic dementia (SD) and a particular type of stroke aphasia, transcortical sensory aphasia (TSA). Both groups had poor comprehension despite fluent speech and good repetition allowing them to be tested on exactly the same lists. The effect of semantic impairment on ISR has been studied in SD and stroke aphasia previously but investigations of these two groups have given rise to distinct 


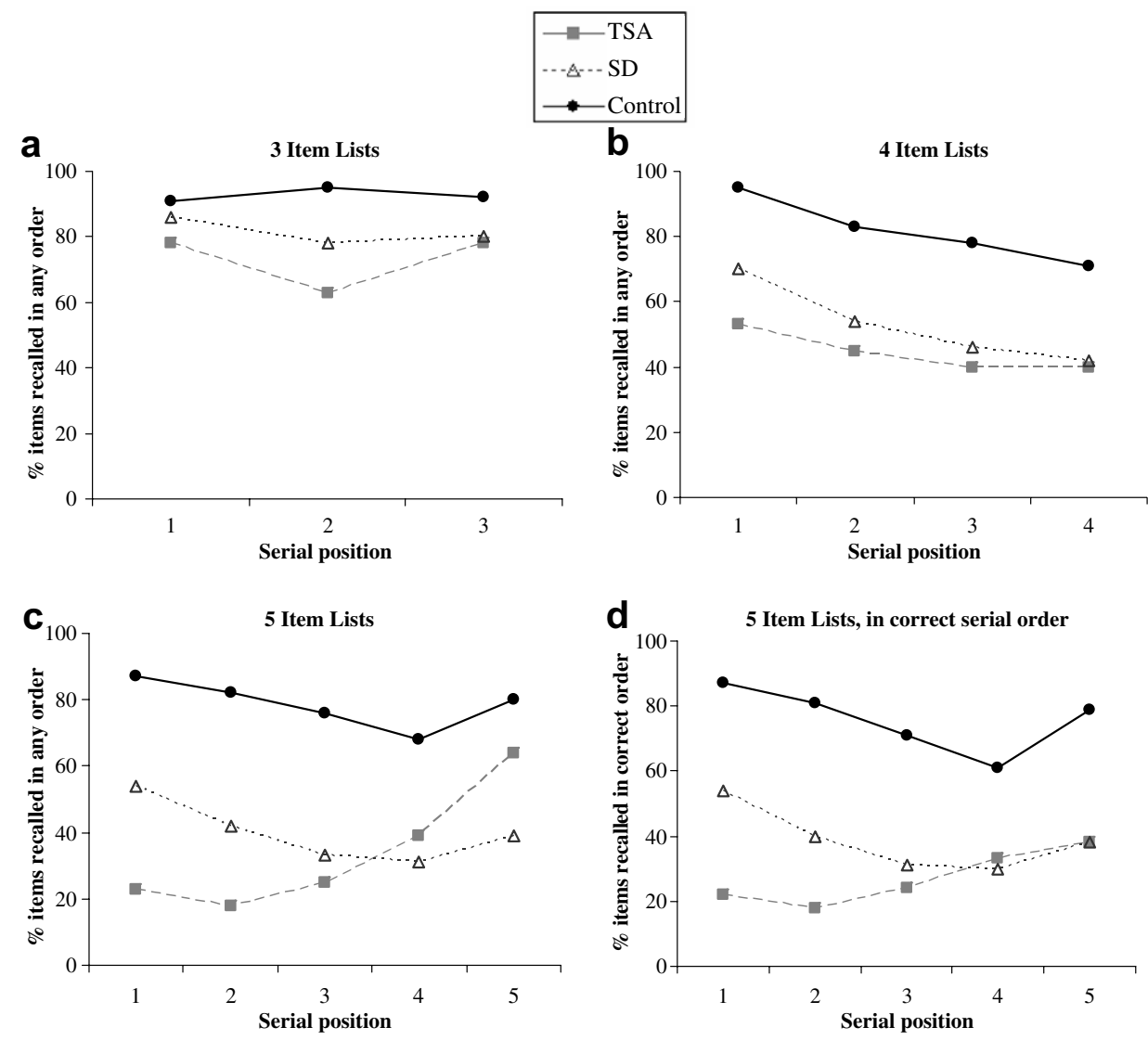

Fig. 5. Serial position curves for lists of three, four and five words recalled in any order (a, b and c) and for five-word lists recalled in the correct serial order $(\mathrm{d})$.

Table 5

Results of $\chi^{2}$ tests comparing recall of initial and final items with middle items for each patient group

\begin{tabular}{|c|c|c|c|c|c|c|}
\hline \multirow[t]{2}{*}{ Group } & \multirow[t]{2}{*}{ List length } & \multicolumn{3}{|c|}{ Proportion of correct responses } & \multirow{2}{*}{$\begin{array}{l}\text { Initial vs. middle } \\
\chi^{2} \text { (primacy) }\end{array}$} & \multirow{2}{*}{$\begin{array}{l}\text { Middle vs. final } \\
\chi^{2} \text { (recency) }\end{array}$} \\
\hline & & Initial & Middle & $\overline{\text { Final }}$ & & \\
\hline \multirow[t]{3}{*}{ SD } & 3 & .86 & .78 & .80 & 1.09 & .06 \\
\hline & 4 & .70 & .50 & .42 & $4.17^{*}$ & .64 \\
\hline & 5 & .54 & .35 & .39 & $14.6^{* *}$ & .69 \\
\hline \multirow[t]{3}{*}{ TSA } & 3 & .78 & .63 & .73 & 2.14 & .91 \\
\hline & 4 & .53 & .48 & .40 & .20 & .46 \\
\hline & 5 & .23 & .28 & .64 & 1.07 & $42.4^{* *}$ \\
\hline \multirow[t]{3}{*}{ Control } & 3 & .91 & .95 & .92 & 1.23 & .74 \\
\hline & 4 & .95 & .81 & .72 & $10.1^{* *}$ & 2.31 \\
\hline & 5 & .87 & .75 & .80 & $12.8^{* *}$ & 2.03 \\
\hline
\end{tabular}

Responses were scored correct if recalled in any order. Middle values were calculated as mean recall for central serial positions in lists of four and five items.

** $\begin{aligned}{ }^{*} & <.05 \\ & <<.001\end{aligned}$

theories about the way in which long-term linguistic knowledge contributes to verbal STM (e.g., the IA model of Martin \& Saffran, 1997; and the semantic binding hypothesis of Patterson et al., 1994). When the results of these earlier studies are put side by side, it appears that semantic deficits in SD and stroke aphasia might have different consequences for ISR, although differences in methodology, specifically the list lengths used, might also explain these discrepancies. The present research demonstrates that there are genuine differences 


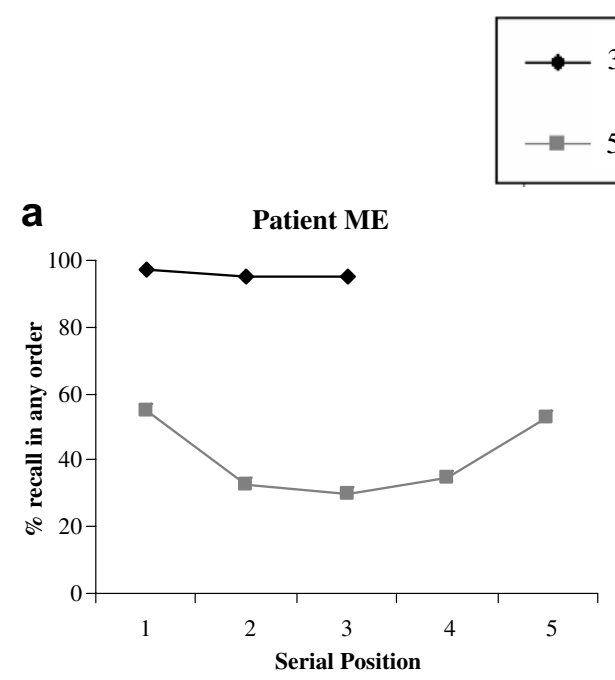

-world lists - 5-world lists
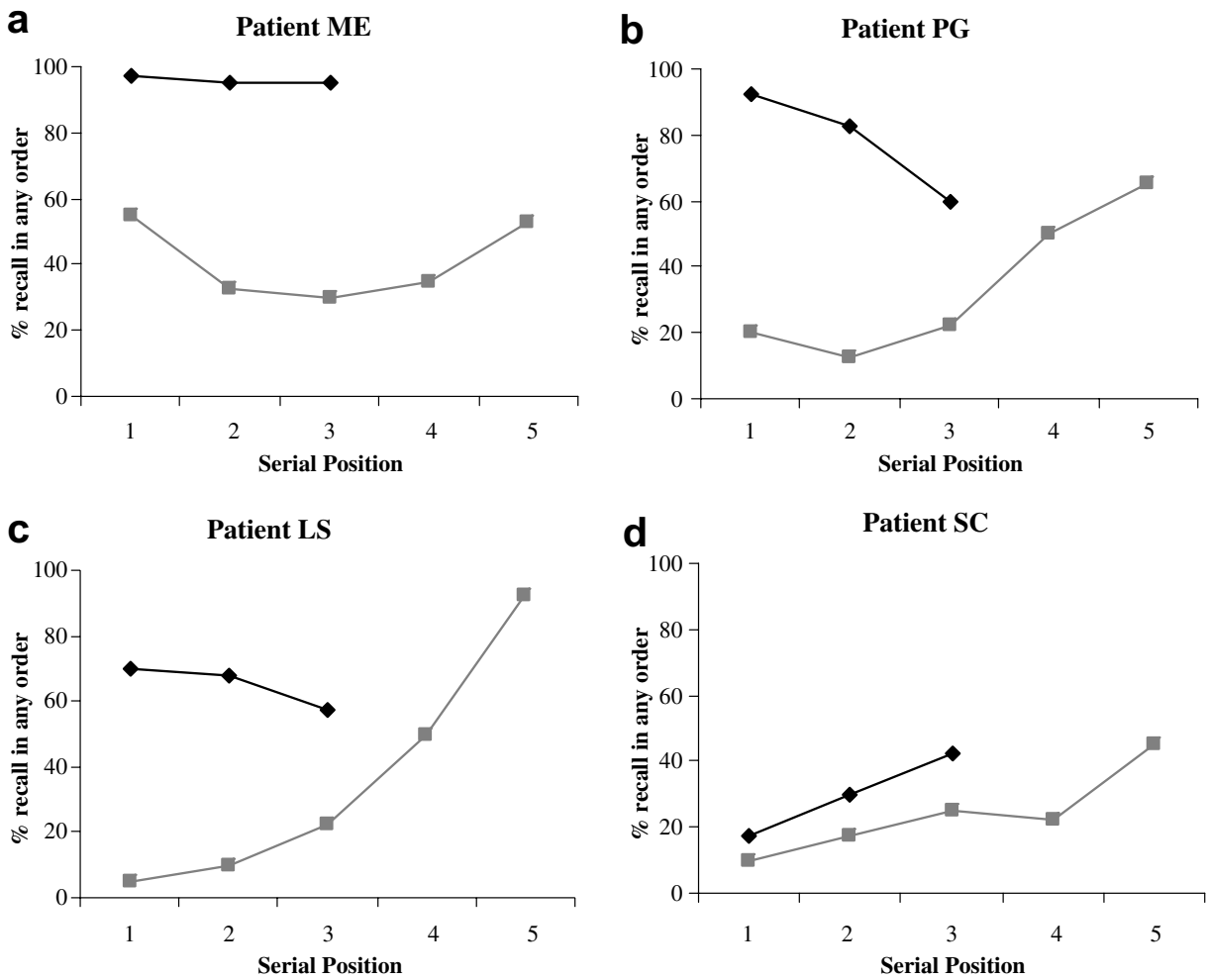

Fig. 6. Serial position curves for each TSA patient on three- and five-word lists.

between SD and TSA patients which are not accommodated by current neuropsychological models of STM. We propose that while the STM impairment in SD reflects a loss of semantic knowledge, the deficit in TSA follows damage to executive control processes that help to direct activation towards task-relevant semantic and linguistic representations, and away from their competitors. We also looked at the extent to which ISR was reliably impaired in TSA. Theoretical frameworks such as the IA model (Martin \& Saffran, 1997) suggest that semantic impairment should affect verbal STM in much the same way in different aphasic individuals. However, we found that there was considerable variation within the TSA group. These findings can be interpreted within a theoretical framework that incorporates information about lesion location.

\section{Summary of results}

Both the SD and TSA patients showed preserved effects of phonological similarity but reduced effects of lexicality in ISR, in line with their similar neuropsycho- logical profiles. The phonological similarity effect has long been associated with phonological coding and rehearsal processes in verbal STM (Conrad, 1964; Conrad \& Hull, 1964), suggesting that both patient groups utilised a phonological code in ISR in a relatively normal way. In contrast, the lexicality effect is thought to reflect the normal contribution of lexical and semantic knowledge to verbal STM (e.g. Hulme et al., 1991). Both patient groups showed a reduced advantage for word over nonword recall suggesting that their semantic deficits disrupted their ability to use word knowledge to support ISR. This deficit was more pronounced for the SD group.

There were four important differences between the patient groups: (1) The SD patients made a large number of phoneme migration errors, in which phonemes from different list items recombined in recall to form new words or neologisms. Phoneme migrations were less common for the TSA group, although they did occur at a higher rate than in controls. (2) The SD patients showed exaggerated effects of both word frequency and imageability in ISR, whereas the TSA patients resembled controls. (3) The SD patients showed normal serial position effects; they 
showed better recall of the initial items in the lists. In contrast, the TSA patients were substantially more likely to recall the final list items, especially for longer lists (although this effect was especially strong for LS and PG). (4) On longer lists, the TSA patients also showed a tendency to recall whole items out of serial order although they were able to repeat items in the correct order at shorter list lengths. The pattern of strong recency coupled with extremely poor recall of initial list items apparently emerged because the TSA patients often reported the final list items first, following this with words from earlier in the list (again, this tendency was strongest for LS and PG). Several of these findings-for example, the differences in frequency/imageability and serial position effects - replicate those observed in earlier studies (see Table 1).

Despite these findings of significant group differences between the SD and TSA patients, there was also considerable variation within the TSA group. ME and SC were more SD-like than the other TSA patients. They showed significant frequency effects in ISR and ME also showed a significant effect of imageability. In addition, these two patients did not show such a pronounced tendency to reproduce the final list item first. These differences (a) between patients with SD and TSA and (b) within the TSA group might be explicable in terms of the specific contributions of different cortical regions to semantic memory and STM.

\section{Theoretical framework}

We propose that the verbal STM impairment in SD and TSA mirrors the nature of the semantic deficit in these conditions. Comprehension problems in SD and TSA are associated with diverse areas of brain damage: SD patients have focal atrophy of the inferior, anterior temporal lobes bilaterally (Mummery et al., 2000; Nestor et al., 2006), while TSA results from lesions to either temporoparietal or prefrontal regions in the left hemisphere (e.g., Berthier, 2001). As noted in the Introduction, a recent study that included the five TSA patients examined here suggested that the nature of the semantic impairment was qualitatively different in SD and stroke aphasia (Jefferies \& Lambon Ralph, 2006). The SD patients showed evidence of degraded semantic representations (in line with the view that the anterior temporal lobes form a store of amodal semantic knowledge; Rogers et al., 2004). The semantic aphasia patients, on the other hand, showed deregulated semantic cognition: they appeared to retain semantic knowledge that they were unable to use appropriately in particular tasks, suggesting that they had difficulty shaping their semantic processing to make it appropriate for the context.

This difference in the nature of the semantic deficit in SD and TSA can explain the pattern of verbal STM impairment in both groups. SD patients make more phoneme migration errors than TSA patients because semantic knowledge stored in the anterior temporal lobes becomes degraded in SD but not TSA. This disrupts the coherence of word forms in ISR. According to the "semantic binding" hypothesis, there are strong bi-directional connections between semantics and phonology that arise because representations pertaining to the same concept are activated together during language production/comprehension (Patterson et al., 1994). As a result, activation within the semantic system encourages the segments of words to be recalled together as a coherent item. The semantic representations that give rise to this constraint are eroded in SD, allowing the phonological elements of different words to become mixed up in ISR tasks. In contrast, the store of semantic information is relatively intact in TSA, and so the phonological system is still able to settle on coherent lexical attractors. However, poor semantic control in TSA may disrupt the semantic contribution to ISR to some extent because two of the TSA patients did produce more phoneme migrations than the controls and the group as a whole showed some reduction in the size of the lexicality effect.

Similarly, frequency and imageability effects in ISR may be exaggerated in patients with SD but not TSA because SD produces damage to semantic representations that is graded by frequency/imageability, whereas TSA does not. Less frequent/familiar concepts may be more susceptible to degradation in SD because they form weaker representations in the semantic system to begin with and/ or because they are encountered less often during the course of this progressive disease (Lambon Ralph, Graham, Ellis, \& Hodges, 1998; Rogers et al., 2004). Similarly, highly imageable words/concepts are thought to possess more semantic features (Plaut \& Shallice, 1991), which may make these representations more resistant to damage. Consequently, ISR will be relatively normal for more frequent/imageable words which are understood comparatively well by SD patients, whereas infrequent/abstract words will receive inadequate support from the semantic system and be recalled poorly in this patient group.

Features of the verbal STM impairment that are specific to TSA - such as poor recall of the initial list items, strong recency effects and a tendency to produce items out of serial order - might reflect difficulty in managing competing representations within the list of items to be recalled (Jefferies et al., 2007). In ISR, recently presented items are likely to be more active than items from the beginning of lists because there has been less time for these items to decay, and yet the task requires items to be recalled in serial order. An inability to resolve competition between activated representations in line with the task demands could therefore lead to the misordering of items, with the most recently presented words being selected for recall first. This deficit would produce strong recency effects (at least when order information is discarded) and poor recall of the primacy portion of the serial posi- 
tion curve, in line with our findings for TSA. We did not observe this pattern for shorter lists of items, perhaps because the phonology of the initial items was less substantially decayed and thus less vulnerable to competition from the final list items. A similar hypothesis was proposed by Martin and Lesch (1996): they described an aphasic patient with a semantic STM deficit who showed a tendency to recall the final list items first and suggested that this might result from excessive interference between items, in line with the view that poor inhibition lies at the heart of this disorder (Hamilton \& Martin, 2005). In contrast, patients with SD do not have poor executive control over semantic/linguistic activation, and thus are able to direct their recall efforts appropriately towards phonological segments presented at the beginnings of lists.

\section{Explaining individual variation within TSA}

Although all of the TSA patients in the current study showed evidence of deregulated semantic cognition, this was true to varying degrees. We reanalysed the data from Jefferies and Lambon Ralph (2006) and Jefferies et al. (2007) to explore individual differences in the nature of the semantic impairment in TSA. Two patients, $\mathrm{ME}$ and SC, who were more SD-like in their ISR performance, were also more similar to the SD patients in some aspects of their semantic impairment.

(1) Jefferies and Lambon Ralph (2006) found that although SD patients showed substantial consistency between different semantic tasks that utilised the same concepts, comprehension-impaired stroke aphasic patients did not. Turning to the TSA patients in the current study, ME/SC showed occasional consistency across different types of semantic tasks whereas PG/LS did not. Data were available for the following tasks that utilised the same set of 64 items: the Camel and Cactus Test of semantic association, presented as either pictures (CCTp) or words (CCTw), word-picture matching (WPM), and sorting picture cards according to semantic category (Sort). Five contingency coefficients were calculated (i.e., consistency between CCTp-WPM; CCTp-Sort; CCTw-WPM; CCTw-Sort; WPM-Sort). ME showed a significant degree of consistency between CCTp and the sorting task ( $C=.24, p=.05)$ although not between the other tasks. SC showed significant consistency between CCTw and sorting $(C=.39, p=.0008)$ and the level of consistency between CCTp and sorting approached significance $(C=.20, p=.1)$. In contrast, PG and LS did not show any evidence of consistency in any of these analyses.

(2) Comprehension in SD patients is highly sensitive to frequency/familiarity, whereas stroke aphasic patients with multimodal semantic impairment show little effect of these variables (Jefferies et al., submit- ted for publication-a; Jefferies \& Lambon Ralph, 2006). ME/SC showed occasional effects of word/ concept frequency in semantic tasks whereas PG/LS did not. We used a median-split method to examine the influence of familiarity/frequency in the five tasks described above (from Jefferies \& Lambon Ralph, 2006). We also examined the effect of word frequency in a synonym judgement task (Jefferies et al. (submitted for publication-a)). SC was significantly affected by frequency in word-picture matching (Fisher exact one-tailed $p=.05)$. ME showed a frequency effect that approached significance in synonym judgement (Fisher exact one-tailed $p=.05$ ). The other patients did not show any tendency towards better performance for high frequency/familiarity items.

(3) SD patients do not show refractory effects in comprehension tasks (i.e. poorer performance when semantically related items are presented repeatedly at a fast rate) whereas stroke aphasic patients with comprehension impairment often do (Jefferies et al., 2007; Warrington \& Cipolotti, 1996). Jefferies et al. (2007) suggested that this might be a consequence of poor semantic control. SC/ME, unlike the other TSA patients in this study, did not show refractory effects on word-picture matching accuracy. However, these two patients did show some sensitivity to refractory effects overall (for ME, in the speed of word-picture matching and for SC in naming accuracy).

These differences between ME/SC and the other TSA patients were relatively subtle: the nature of the semantic impairment for all of the TSA patients was strikingly different from that seen in SD. For example, ME and SC were highly sensitive to phonemic cues in picture naming, as were the other TSA patients. In contrast, the SD patients were largely insensitive to such cues (Jefferies, Patterson, \& Lambon Ralph, submitted for publication-b). Similarly, in the present study, we observed clear and significant differences between SD and TSA in verbal STM tasks as well as some variation within the TSA group. The TSA patients with the most marked deficits of semantic control also showed certain features of the ISR impairment to a greater extent - in particular, a tendency to reproduce the final list item first, resulting in frequent serial order errors and strong recency effects. This variation might be explicable in terms of the location of the TSA patients' lesions (see below).

\section{Overlap with other proposals}

The IA framework put forward by Martin and Saffran (1997) cannot explain the TSA patients' tendency to recall items out of serial order because, as a model of single word production, it lacks a mechanism for representing serial order in lists. In implemented models of STM, this 
sequencing function is often achieved through the formation of associations between list items and a temporal context signal (Brown, Preece, \& Hulme, 2000; Burgess \& Hitch, 1992, 1999; Gupta \& MacWhinney, 1997; Hartley \& Houghton, 1996; Henson, 1998). During list recall, activation within the context units is replayed, allowing the words to be recalled in the correct order. Models of this nature typically work as follows: when list recall is initiated, the first item receives the most activation from the context units, combating the natural decay processes that would otherwise leave the most recently presented item with the highest activation. The strongly activated initial item is selected for production and then inhibited. Following its inhibition, the second item is the most active so is selected for production and recall continues in this way. Failure to apply this mechanism might result in the listorder reversal observed in our TSA patients because in the absence of a context signal, the most active item is likely to be the last to be presented.

This mechanism can underpin memory for serial order but does not motivate a link between STM and cognitive control more generally. This is required for a parsimonious account of our TSA patients' performance because their deficits in both semantic tasks and ISR appeared to be linked to their executive dysfunction. An alternative perspective is that memory for serial order is partly underpinned by a more general cognitive control system that can direct attention to task-relevant representations (see Hazy, Frank, \& O'Reilly, 2006; O'Reilly, Braver, \& Cohen, 1999; O'Reilly \& Soto, 2002). Although in most speech production tasks, the most active phonological representations are selected for output, the relevance of representations in ISR is dependent on the order in which the items were presented. Executive control might play a role in overcoming the well-established tendency to select the most active item for output. This is likely to be especially true for arbitrary sequences of unrelated items..$^{5}$

Martin and Gupta (2004) also suggested that ISR differences between semantically impaired SD and stroke aphasic patients might reflect the nature of the semantic impairment in the two groups. However, they modelled stroke aphasia as rapid decay within semantic-lexical connections and contrasted this with degradation of semantic representations in SD. This might account for the different serial position effects seen in the two groups previously. Although SD patients have impoverished semantic knowledge, some semantic representations will remain intact and will feed activation back to the phonological layer in a normal fashion, benefiting

\footnotetext{
${ }^{5}$ This is not incompatible with the notion that the phonological system can capture some serial order information automatically. Indeed, the TSA patients had good single word repetition and fluent speech production, possibly reflecting this capacity.
}

the recall of early list items according to the IA model. In stroke aphasia, in contrast, pathological decay between the lexical and semantic layers will make these patients more reliant on recent phonological activation. This cannot provide an explanation of our data, however, as the TSA group showed parallel deficits on verbal and nonverbal semantic tasks. We suggest instead that a single difficulty managing competition between activated representations could explain these patients' poor ISR and difficulties on both verbal and non-verbal semantic tasks. Moreover, the rapid decay of target activation highlighted by Martin and Gupta (2004) could be another consequence of poor cognitive control in TSA because a failure to direct attention towards targets and away from competitors would result in the target response becoming rapidly unavailable.

\section{Neuroanatomy}

The TSA patients in the present study had damage to prefrontal and/or temporoparietal regions of the left hemisphere (contrasting with the anterior temporal lobe atrophy seen in SD). Previous studies have shown that TSA patients with these two types of lesions show very similar behavioural deficits (Berthier, 2001). The prefrontal cortex and temporoparietal junction are thought to form a single distributed system underpinning cognitive control, explaining why patients with lesions that encompass these two regions show similar behavioural deficits (see Peers et al., 2005). Prefrontal and temporoparietal cortex show coupled activation during the performance of attentional/executive tasks and are sensitive to manipulations of the executive component of working memory (Collette et al., 2006; Collette et al., 2005, 2002; Crosson et al., 1999; Garavan, Ross, Li, \& Stein, 2000).

However, the present study suggests that there might be some specialisation in this prefrontal-temporoparietal network. The two patients who consistently reversed item order in recall (LS and PG) had lesions that included the left inferior prefrontal cortex, while damage was restricted to left temporoparietal areas in the two patients who did not (SC and ME). Similarly, a previously reported stroke aphasic case who initiated recall with terminal list items had prefrontal damage (Martin $\&$ Lesch, 1996). The left prefrontal cortex may therefore play a particularly critical role in overcoming the proponent tendency to select the most highly activated items for output. Previous work has highlighted the role of this region in selecting between competing responses, in line with this conclusion (Hamilton \& Martin, 2005; Robinson, Shallice, \& Cipolotti, 2005; Schnur, Schwartz, Brecher, \& Hodgson, 2006; Thompson-Schill, D’Esposito, Aguirre, \& Farah, 1997; Thompson-Schill et al., 1998; Wagner, Pare-Blagoev, Clark, \& Poldrack, 2001). 


\section{Conclusions}

We have demonstrated qualitative differences in the impact of semantic impairment on ISR in semantic dementia and transcortical sensory aphasia. Our SD cases showed abnormally large effects of frequency/imageability and made numerous phoneme migration errors in ISR, reflecting their degraded knowledge of lower frequency items. Patients with TSA, on the other hand, displayed normal effects of lexical-semantic variables in ISR and showed large recency effects on longer lists, partly due to a tendency to reverse list order in recall. The TSA patients' difficulties appeared to stem from an inability to resolve competition between lexical-semantic representations, in line with their poor semantic control on other tasks that did not tap short-term memory.

\section{Acknowledgments}

We are indebted to the patients and their carers for their generous assistance with this study. We thank Linda Collier and Claire Slinger for referring some of the patients to us. Sharone Borbe collected some of the control data. We gratefully acknowledge Peter Garrard's assistance with the interpretation of the brain scans and Karen Sage's help with classifying the stroke patients' aphasic syndromes. We thank Nadine Martin for some interesting discussions about this work. The study was supported by an IBSC grant from the NIMH (MH64445), an RCUK fellowship awarded to E. Jefferies and an MRC programme Grant (G0501632).

\section{Appendix A}

Characteristics of stimuli

\begin{tabular}{lllll}
\hline & \multicolumn{2}{c}{ Imageability } & \multicolumn{2}{c}{ Frequency } \\
\hline High frequency & 519.1 & n.s. & 186.1 & $p<.001$ \\
$(N=100)$ & $(86.6)$ & & $(155.6)$ & \\
Low frequency & 522.1 & & 7.8 & \\
$(N=100)$ & $(84.4)$ & & $(8.2)$ & \\
High imageability & 599.5 & $p<.001$ & 89.5 & n.s. \\
$(N=100)$ & $(19.0)$ & & $(138.6)$ & \\
Low imageability & 440.8 & & 104.3 & \\
$(N=100)$ & $(39.6)$ & & $(144.9)$ & \\
\hline
\end{tabular}

Figures show mean for each group (standard deviations in parentheses). n.s., not significant (all $p>.1$ ). Frequency values are from Kucera and Francis (1967); imageability values are from the MRC database (Coltheart, 1981).

\section{References}

Albert, M., Goodglass, H., Helms, N. A., Rubens, A. B., \& Alexander, M. N. (1981). Clinical aspects of dysphasia. New York/Berlin: Springer-Verlag.
Baddeley, A. D. (1966). Short-term memory for word sequences as a function of acoustic, semantic and formal similarity. Quarterly Journal of Experimental Psychology, 18, 362-365.

Baldo, J. V., Dronkers, N. F., Wilkins, D., Ludy, C., Raskin, P., \& Kim, J. Y. (2005). Is problem solving dependent on language?. Brain and Language 92, 240-250.

Berthier, M. L. (2000). Transcortical aphasias. Hove, UK: Psychology Press.

Berthier, M. L. (2001). Unexpected brain-language relationships in aphasia: Evidence from transcortical sensory aphasia associated with frontal lobe lesions. Aphasiology, $15,99-130$.

Bird, H., Lambon Ralph, M. A., Seidenberg, M. S., McClelland, J. L., \& Patterson, K. (2003). Deficits in phonology and past-tense morphology: What's the connection? Journal of Memory and Language, 48, 502-526.

Bourassa, D. C., \& Besner, D. (1994). Beyond the articulatory loop - a semantic contribution to serial order recall of subspan lists. Psychonomic Bulletin \& Review, 1, 122-125.

Bozeat, S., Lambon Ralph, M. A., Patterson, K., Garrard, P., \& Hodges, J. R. (2000). Non-verbal semantic impairment in semantic dementia. Neuropsychologia, 38, 1207-1215.

Brener, R. (1940). An experimental investigation of memory span. Journal of Experimental Psychology, 26, 467-482.

Brown, G., Preece, T., \& Hulme, C. (2000). Oscillator-based memory for serial order. Psychological Review, 107, 127-181.

Burgess, N., \& Hitch, G. J. (1992). Toward a network model of the articulatory loop. Journal of Memory and Language, 31, 429-460.

Burgess, N., \& Hitch, G. J. (1999). Memory for serial order: A network model of the phonological loop and its timing. Psychological Review, 106, 551-581.

Chertkow, H., Bub, D., Deaudon, C., \& Whitehead, V. (1997). On the status of object concepts in aphasia. Brain and Language, 58, 203-232.

Collette, F., Hogge, M., Salmon, E., \& Van der Linden, M. (2006). Exploration of the neural substrates of executive functioning by neuroimaging. Neuroscience, 139, 209-221.

Collette, F., Olivier, L., Van der Linden, M., Laureys, S., Delfiore, G., Luxen, A., et al. (2005). Involvement of both prefrontal and inferior parietal cortex in dual-task performance. Cognitive Brain Research, 24, 237-251.

Collette, F., \& Van der Linden, M. (2002). Brain imaging of the central executive component of working memory. Neuroscience and Biobehavioral Reviews, 26, 105-125.

Coltheart, M. (1981). The MRC Psycholinguistic Database. Quarterly Journal of Experimental Psychology Section A: Human Experimental Psychology, 33, 497-505.

Conrad, R. (1964). Acoustic confusion in immediate memory. British Journal of Psychology, 55, 75-84.

Conrad, R., \& Hull, A. J. (1964). Information, acoustic confusion and memory span. British Journal of Psychology, $55,432-439$.

Crawford, J. R., \& Garthwaite, P. H. (2002). Investigation of the single case in neuropsychology: Confidence limits on the abnormality of test scores and test score differences. Neuropsychologia, 40, 1196-1208.

Crosson, B., Rao, S. M., Woodley, S. J., Rosen, A. C., Bobholz, J. A., Mayer, A., et al. (1999). Mapping of semantic, phonological, and orthographic verbal working 
memory in normal adults with functional magnetic resonance imaging. Neuropsychology, 13, 171-187.

Dell, G. S., \& O'Seaghda, P. G. (1992). Stages of lexical access in language production. Cognition, 42, 287-314.

De Renzi, E., Faglioni, P., Scotti, G., \& Spinnler, H. (1972). Impairment in associating colour to form, concomitant with aphasia. Brain, 95, 293-304.

Forde, E. M. E., \& Humphreys, G. W. (2002). The role of semantic knowledge in short-term memory. Neurocase, 8 , $13-27$.

Funnell, E. (1995). Objects and properties: A study of the breakdown of semantic memory. Memory, 3, 497-518.

Gainotti, G., Miceli, G., \& Caltagirone, C. (1979). The relationship between conceptual and semantic-lexical disorders in aphasia. International Journal of Neuroscience, $10,45-50$.

Gainotti, G., Silveri, M. C., Villa, G., \& Caltagirone, C. (1983). Drawing objects from memory in aphasia. Brain, 106, 613-622.

Garavan, H., Ross, T. J., Li, S. J., \& Stein, E. A. (2000). A parametric manipulation of central executive functioning. Cerebral Cortex, 10, 585-592.

Gathercole, S. E., Pickering, S. J., Hall, M., \& Peaker, S. M. (2001). Dissociable lexical and phonological influences on serial recognition and serial recall. The Quarterly Journal of Experimental Psychology, 54A, 1-30.

Goodglass, H. (1983). The assessment of aphasia and related disorders (2nd ed.). Philadelphia: Lea \& Febiger.

Gregg, V. H., Freedman, C. M., \& Smith, D. K. (1989). Word frequency, articulatory suppression and memory span. British Journal of Psychology, 80, 363-374.

Gupta, P., \& MacWhinney, B. (1997). Vocabulary acquisition and verbal short-term memory: Computational and neural bases. Brain and Language, 59, 267-333.

Hamilton, A. C., \& Martin, R. C. (2005). Dissociations among tasks involving inhibition: A single-case study. Cognitive, Affective, \& Behavioral Neuroscience, 5, 1-13.

Hartley, T., \& Houghton, G. (1996). A linguistically constrained model of short-term memory for nonwords. Journal of Memory and Language, 35, 1-31.

Hazy, T. E., Frank, M. J., \& O’Reilly, R. C. (2006). Banishing the homunculus: Making working memory work. Neuroscience, 139, 105-118.

Henson, R. N. A. (1998). Short-term memory for serial order: The start-end model. Cognitive Psychology, 36, 73-137.

Hodges, J. R., Patterson, K., Oxbury, S., \& Funnell, E. (1992). Semantic dementia: Progressive fluent aphasia with temporal lobe atrophy. Brain, 115, 1783-1806.

Howard, D., \& Patterson, K. (1992). Pyramids and palm trees: $A$ test of semantic access from pictures and words. Bury St. Edmunds, UK: Thames Valley Test Company.

Hulme, C., Maughan, S., \& Brown, G. D. A. (1991). Memory for familiar and unfamiliar words: Evidence for a long-term memory contribution to short-term memory span. Journal of Memory and Language, 30, 685-701.

Hulme, C., Roodenrys, S., Schweickert, R., Brown, G. D. A., Martin, S., \& Stuart, G. (1997). Word-frequency effects on short-term memory tasks: Evidence for a redintegration process in immediate serial recall. Journal of Experimental Psychology: Learning, Memory and Cognition, 23, 1217-1232.
Jefferies, E., Baker, S. S., Doran, M., \& Lambon Ralph, M. A. (2007). Refractory effects in semantically impaired stroke aphasic patients. Neuropsychologia, 45, 1065-1079.

Jefferies, E., Crisp, J., \& Lambon Ralph, M. A. (2006). The impact of phonological or semantic impairment on delayed auditory repetition: Evidence from stroke aphasia and semantic dementia. Aphasiology, 20, 963-993.

Jefferies, E., Corbett, F., Hopper, S., \& Lambon Ralph, M. A. (submitted for publication-a). Frequency and imageability effects in comprehension: Dissociations between semantic dementia and semantic aphasia.

Jefferies, E., Frankish, C., \& Lambon Ralph, M. A. (2006). Lexical and semantic binding in verbal shortterm memory. Journal of Memory and Language, 54, $81-98$.

Jefferies, E., Jones, R., Bateman, D., \& Lambon Ralph, M. A. (2004). When does word meaning affect immediate serial recall in semantic dementia? Cognitive, Affective and Behavioral Neuroscience, 4, 20-42.

Jefferies, E., Jones, R. W., Bateman, D., \& Lambon Ralph, M. A. (2005). A semantic contribution to nonword recall? Evidence for intact phonological processes in semantic dementia. Cognitive Neuropsychology, 22, 183-212.

Jefferies, E., \& Lambon Ralph, M. A. (2006). Semantic impairment in stroke aphasia vs. semantic dementia: A case-series comparison. Brain, 129, 2132-2147.

Jefferies, E., Patterson, K., Bateman, D., Jones, R., \& Lambon Ralph, M. A. (2006). The natural history of "pure" latestage semantic dementia. Neurocase, 12, 1-14.

Jefferies, E., Patterson, K., Jones, R. W., Bateman, D., \& Lambon Ralph, M. A. (2004a). A category-specific advantage for numbers in verbal short-term memory: Evidence from semantic dementia. Neuropsychologia, 42, 639-660.

Jefferies, E., Patterson, K., \& Lambon Ralph, M. A. (submitted for publication-b). Deficits of knowledge vs. executive control in semantic cognition: Insights from cued naming.

Jones, G. V. (1985). Deep dyslexia, imageability and ease of prediction. Brain and Language, 24, 1-19.

Kay, J., Lesser, R., \& Coltheart, M. (1992). Psycholinguistic Assessments of Language Processing in Aphasia (PALPA). Hove, UK: Lawrence Erlbaum Associates.

Knott, R., Patterson, K., \& Hodges, J. R. (1997). Lexical and semantic binding effects in short-term memory: evidence from semantic dementia. Cognitive Neuropsychology, 14, $1165-1216$.

Knott, R., Patterson, K., \& Hodges, J. R. (2000). The role of speech production in auditory-verbal short-term memory: evidence from progressive fluent aphasia. Neuropsychologia, $38,125-142$.

Kucera, H., \& Francis, W. N. (1967). Computational analysis of present-day American English. Providence, RI: Brown University Press.

Lambon Ralph, M. A., Graham, K. S., Ellis, A. W., \& Hodges, J. R. (1998). Naming in semantic dementia-what matters? Neuropsychologia, 36, 775-784.

Majerus, S., Norris, D., \& Patterson, K. (2007). What does a patient with semantic dementia remember in verbal shortterm memory? Order and sound but not words. Cognitive Neuropsychology, 24, 131-151. 
Martin, N., \& Ayala, J. (2004). Measurements of auditoryverbal STM span in aphasia: Effects of item, task and lexical impairment. Brain and Language, 89, 464-483.

Martin, N., Ayala, J., \& Saffran, E. M. (2002). Lexical influences on serial position effects in verbal STM span in aphasia. Brain and Language, 83, 92-95.

Martin, N., \& Gupta, P. (2004). Exploring the relationship between word processing and verbal short-term memory: Evidence from associations and dissociations. Cognitive Neuropsychology, 21, 213-228.

Martin, R. C., \& Lesch, M. (1996). Associations and dissociations between language impairment and list recall: Implications for models of STM. In S. E. Gathercole (Ed.), Models of short-term memory (pp. 149-178). East Sussex, UK: Psychology Press.

Martin, R. C., Lesch, M. F., \& Bartha, M. C. (1999). Independence of input and output phonology in word processing and short-term memory. Journal of Memory and Language, 41, 3-29.

Martin, N., \& Saffran, E. (1990). Repetition and verbal STM in transcortical sensory aphasia: A case study. Brain and Language, 39, 254-288.

Martin, N., \& Saffran, E. M. (1997). Language and auditoryverbal short-term memory impairments: Evidence for common underlying processes. Cognitive Neuropsychology, 14, 641-682.

Martin, R. C., Shelton, J., \& Yaffee, L. S. (1994). Language processing and working memory: Neuropsychological evidence for separate phonological and semantic capacities. Journal of Memory and Language, 33, 83-111.

McCarthy, R. A., \& Warrington, E. K. (2001). Repeating without semantics: Surface dysphasia? Neurocase, 7, 77-87.

Mummery, C. J., Patterson, K., Price, C. J., Ashburner, J., Frackowiak, R. S. J., \& Hodges, J. R. (2000). A voxel-based morphometry study of semantic dementia: Relationship between temporal lobe atrophy and semantic memory. Annals of Neurology, 47, 36-45.

Nestor, P. J., Fryer, T. D., \& Hodges, J. R. (2006). Declarative memory impairments in Alzheimer's disease and semantic dementia. Neuroimage, 30, 1010-1020.

O'Reilly, R. C., Braver, T. S., \& Cohen, J. D. (1999). A biologically based computational model of working memory. In A. Miyake \& S. Shah (Eds.), Models of working memory: Mechanisms of active maintenance and executive control (pp. 375-411). New York: Cambridge University Press.

O'Reilly, R. C., \& Soto, R. (2002). A Model of the Phonological Loop: Generalization and Binding. In T. G. Dietterich, S. Becker, \& Z. Ghahramani (Eds.), Advances in Neural Information Processing Systems (NIPS) 14. Cambridge, MA: MIT Press.

Patterson, K., Graham, N., \& Hodges, J. R. (1994). The impact of semantic memory loss on phonological representations. Journal of Cognitive Neuroscience, 6, 57-69.

Patterson, K., \& Marcel, A. J. (1992). Phonological ALEXIA or PHONOLOGICAL alexia? In J. Alegria, D. Holender, J. Junca de Morais, \& M. Radeau (Eds.), Analytic approaches to human cognition (pp. 259-274). Amsterdam: Elsevier.

Peers, P. V., Ludwig, C. J. H., Rorden, C., Cusack, R., Bonfiglioli, C., Bundesen, C., et al. (2005). Attentional functions of parietal and frontal cortex. Cerebral Cortex, 15, 1469-1484.

Plaut, D. C., \& Shallice, T. (1991). Deep dyslexia: A case study in connectionist neuropsychology. Cognitive Neuropsychology, 10, 377-500.

Poirier, M., \& Saint-Aubin, J. (1995). Memory for related and unrelated words: Further evidence on the influence of semantic factors in immediate serial recall. The Quarterly Journal of Experimental Psychology, 48 A, 384-404.

Reilly, J., Martin, N., \& Grossman, M. (2005). Verbal learning in semantic dementia: Is repetition priming a useful strategy? Aphasiology, 19, 329-339.

Robinson, G., Shallice, T., \& Cipolotti, L. (2005). A failure of high level verbal response selection in progressive dynamic aphasia. Cognitive Neuropsychology, 22, 661-694.

Rogers, T. T., Lambon Ralph, M. A., Garrard, P., Bozeat, S., McClelland, J. L., Hodges, J. R., et al. (2004). The structure and deterioration of semantic memory: A neuropsychological and computational investigation. Psychological Review, 111, 205-235.

Roodenrys, S., \& Quinlan, P. T. (2000). The effects of stimulus set size and word frequency on verbal serial recall. Memory, $8,71-78$.

Saint-Aubin, J., \& Poirier, M. (1999). Semantic similarity and immediate serial recall: Is there a detrimental effect on order information? Quarterly Journal of Experimental Psychology, 52A, 367-394.

Saygin, A. P., Dick, F., Wilson, S. M., Dronkers, N., \& Bates, E. (2003). Neural resources for processing language and environmental sounds. Brain, 126, 928-945.

Schnur, T. T., Schwartz, M. F., Brecher, A., \& Hodgson, C. (2006). Semantic interference during blocked-cyclic naming: Evidence from aphasia. Journal of Memory and Language, 54, 199-227.

Snodgrass, J. G., \& Vanderwart, M. (1980). Standardized set of 260 pictures - norms for name agreement, image agreement, familiarity, and visual complexity. Journal of Experimental Psychology-Human Learning and Memory, 6, 174-215.

Snowden, J. S., Goulding, P. J., \& Neary, D. (1989). Semantic dementia: A form of circumscribed cerebral atrophy. Behavioural Neurology, 2, 167-182.

Spinnler, H., \& Vignolo, L. A. (1966). Impaired recognition of meaningful sounds in aphasia. Cortex, 2, 337-348.

Thompson-Schill, S. L., D’Esposito, M., Aguirre, G. K., \& Farah, M. J. (1997). Role of left inferior prefrontal cortex in retrieval of semantic knowledge: A reevaluation. Proceedings of the National Academy of Sciences of the United States of America, 94, 14792-14797.

Thompson-Schill, S. L., Swick, D., Farah, M. J., D’Esposito, M., Kan, I. P., \& Knight, R. T. (1998). Verb generation in patients with focal frontal lesions: A neuropsychological test of neuroimaging findings. Proceedings of the National Academy of Sciences of the United States of America, 95, 15855-15860.

Wagner, A. D., Pare-Blagoev, E. J., Clark, J., \& Poldrack, R. A. (2001). Recovering meaning: Left prefrontal cortex guides controlled semantic retrieval. Neuron, 31, 329-338.

Walker, I., \& Hulme, C. (1999). Concrete words are easier to recall than abstract words: Evidence for a semantic contribution to short-term serial recall. Journal of Experimental 
Psychology: Learning, Memory and Cognition, 25, 1256-1271Psychological Corporation.

Warrington, E. K., \& Cipolotti, L. (1996). Word comprehension: The distinction between refractory and storage impairments. Brain, 119, 611-625.

Watkins, O. C., \& Watkins, M. J. (1977). Serial recall and the modality effect: Effects of word frequency. Journal of
Experimental Psychology: Human Learning and Memory, 3, 712-718.

Wechsler, D. (1987). Wechsler Memory Scale-Revised (WMS$R)$. New York: Psychological Corporation.

Wiener, D. A., Connor, L. T., \& Obler, L. K. (2004). Inhibition and auditory comprehension in Wernicke's aphasia. Aphasiology, 18, 599-609. 